\title{
Frictional Heating in the Strip-Foundation Tribosystem
}

\author{
Aleksander Yevtushenko and Michal Kuciej \\ Bialystok University of Technology \\ Poland
}

\section{Introduction}

During the process of braking the pad is pressed to the disc. As a result of friction on the contact surface the kinetic energy transforms into heat. Elements of brakes are heated and, hence, the conditions of operation of the friction pads become less favorable: their wear intensifies and the friction coefficient decreases, which may lead to emergency situations (Fasekas, 1953; Ho at al., 1974). Thus, the correct calculation of temperature is one of the most important challenges in the design of brakes (YBo at al., 2002; Chichinadze at al., 1979; Balakin and Sergienko, 1999).

The heat conduction problems of friction are nowadays formulated in two variants. In the first one, the elements of friction couple are considered separately and the heat flow intensities are set on each of the contact surfaces in such a way that their sum equals the specific power of friction (Ling, 1973; Burton, 1975; Archard and Rowntree, 1988; Kannel and Barber, 1989; Yevtushenko at al., 1995). For this purpose, the heat participation factor is introduced which is found experimentally or by empirical formulas (Block, 1937; Jaeger, 1932). It is the statement that gives the solution of the heat conduction problem of friction for the foundation with a composite strip (Matysiak at al., 2004; Yevtushenko at al., 2007a) and the heat conduction problem for the braking of a massive body coated with either a homogeneous (Evtushenko at al., 2005) or a composite (Matysiak at al., 2007) strip. The thermoelastic state resulted from the heating of the piecewise-homogeneous body consisting of a semi-infinite foundation and a strip by the heat pulse of a finite duration is studied in article (Yevtushenko at al., 2005).

Another variant of the statement of heat conduction problems of friction is based on the simultaneous solution of the heat conduction equations for both friction elements followed by the determination of the heat flows intensities on their heating (Cameron at al., 1965; Barber and Comminou, 1989; Olesiak at al., 1997; Evtushenko and Pyr'ev, 2000; Bauzin and Laraqi, 2004). In such statement the problems of transient frictional heating in cold rolling of metals (Matysiak at al., 1998), the heat transfer in friction welding of cylindrical rods with different diameters (Kahveci at al., 2005) and the fast-moving heating on the external surface of the ring due to friction of two rotating pins (Yevtushenko and Tolstoj-Sienkiewicz, 2006) were analyzed. The analytical solution of a boundary-value problem of heat conduction for tribosystem, consisting of the homogeneous semi-space, sliding uniformly on a surface of the strip deposited on a semi-infinite substrate, was obtained in paper (Yevtushenko and Kuciej, 2009a). 
Heat conditions on the friction surface of two elastic bodies were formulated in the contemporary form for the first time by F. Ling (1959). He thought that in every point on the contact surfaces, the temperature of bodies being in contact is equal. Whereas the sum of heat flow intensities directed from friction surface to interior of each elements equals the power of friction (the perfect thermal contact is assumed). Such approach to statement of the contact problem with heat generation due to friction taken under consideration, assumes temperature dependence only on thermo-physical properties of friction pair materials, on cooling conditions and on power of friction.

It is well known that on friction processes, wear and heat generation, the significant influence have topographical parameters of surfaces being in contact and characteristics of so called "third body" - thin subsurface strips having properties partly physical and mechanical which differ from friction pair materials properties (Johnson, 1987; Godet, 1990; Iordanoff at al., 2002). Thermal contact of bodies, when temperature on the friction surface has jumped, is called imperfect (Barber and Comminou, 1989). The theoretical model of calculation of the contact characteristics (the temperature, the wear and the speed of sliding) during braking is based usually on the assumption that the friction elements can be treated as semi-spaces. The solution to the problem of heat generation during braking with a uniform retardation for two semi-spaces in perfect contact was obtained in articles (Grylytskyy, 1996; Yevtushenko at al., 1999), and in imperfect contact - in articles (Levitskij and Onyshkievich, 1999; Nosko and Nosko, 2006). The contact temperature, the value of wear, and the speed of sliding during braking, for general experimental dependences of the coefficients of friction and wear on the temperature were studied in article (Olesiak at al., 1997).

Solutions to the transient heat conduction problems for a massive body (the semi-space) coated with either a homogeneous, or a composite strip were suggested in articles (Yevtushenko at al., 2007a; Matysiak at al., 2007; Evtushenko at al., 2005). The thermal stresses resulted from the heating of the piecewise-homogeneous body, consisting of a semiinfinite foundation and a strip, by the heat pulse of a finite duration was studied in article (Yevtushenko at al., 2007b).

\section{Evolution of the contact pressure and sliding speed during braking}

The nature of change in time of the specific load on the nominal contact depends on a loading system (pneumatic, hydraulic, mechanical, electromagnetic), and a change of loading during braking (pulse braking, anti-lock braking system, automatic stability control system, etc.). Generally, the evolution of the pressure and movement during braking can be described by the equations (Chichinadze at al., 1979; Ginsburg and Chichinadze, 1978; Chichinadze, 1967)

$$
\begin{gathered}
p(\tau)=p_{0} p^{*}(\tau), p^{*}(\tau)=\left(1-e^{-\tau / \tau_{m}}\right)\left[1+a \sin \left(\omega_{p} \tau\right)\right], 0 \leq \tau \leq \tau_{s}, a \geq 0, \omega_{p}>0, \\
\frac{2 W_{0}}{V_{0}^{2}} \frac{d V(\tau)}{d \tau}=-f p(\tau) A_{k}, 0 \leq \tau \leq \tau_{s}, V(0)=V_{0} . \\
\tau=\frac{k_{s} t}{d^{2}}, \tau_{s}=\frac{k_{s} t_{s}}{d^{2}}
\end{gathered}
$$


where $t$ - time; $t_{s}$ - braking time; $p$ - pressure; $p_{0}$ - nominal pressure; $f$ - coefficient of friction; $k_{s}$ - thermal diffusivity of a strip; $A_{k}$ - nominal contact area; $d$ - strip thickness; $W_{0}$ - the initial kinetic energy; $V$ - sliding speed; $V_{0}$ - initial sliding speed; $a$ - amplitude of pressure oscillations; $\omega_{p}$ - frequency of pressure oscillations.

Substituting the pressure of (2.1) to the right side of the equation (2.2), after integration we obtain

$$
V^{*}(\tau)=V_{1}^{*}(\tau)-\frac{a}{\tau_{s}^{0}} V_{2}^{*}(\tau), 0 \leq \tau \leq \tau_{s}
$$

where

$$
\begin{gathered}
V_{1}^{*}(\tau)=1-\frac{\tau}{\tau_{s}^{0}}+\frac{\tau_{m}}{\tau_{s}^{0}}\left(1-e^{-\tau / \tau_{m}}\right), \\
V_{2}^{*}(\tau)=\frac{1}{\omega_{p}}\left[1-\cos \left(\omega_{p} \tau\right)\right]+\frac{1}{\tau_{m}^{-2}+\omega_{p}^{2}}\left\{\left[\tau_{m}^{-1} \sin \left(\omega_{p} \tau\right)+\omega_{p} \cos \left(\omega_{p} \tau\right)\right] e^{-\tau / \tau_{m}}-\omega_{p}\right\}, \\
\tau_{s}^{0}=\frac{k_{s} t_{s}^{0}}{d^{2}}, \tau_{m}=\frac{k_{s} t_{m}}{d^{2}},
\end{gathered}
$$

$t_{m}$ - duration of the increase of the loading from zero to maximum value $p_{0}, t_{s}^{0}$ - duration of braking in the case of constant pressure.

At the stop time moment $\tau=\tau_{s}$ from equations (2.4)-(2.7) we find the non-linear equation of the dimensionless time of braking $\tau_{s}$ in the form

$$
\tau_{s}^{0} V_{1}^{*}\left(\tau_{s}\right)=a V_{2}^{*}\left(\tau_{s}\right)
$$

If the increase in pressure is monotonically without oscillations $(a=0)$, then the equations (2.1), (2.4) and (2.8) take the form

$$
p^{*}(\tau)=1-e^{-\tau / \tau_{m}}, V^{*}(\tau)=1-\frac{\tau}{\tau_{s}^{0}}+\frac{\tau_{m}}{\tau_{s}^{0}}\left(1-e^{-\tau / \tau_{m}}\right), 0 \leq \tau \leq \tau_{s}
$$

If pressure reaches its maximal value $p_{0}$ immediately $\left(\tau_{m} \rightarrow 0\right)$, then from equations (2.9) it follows that

$$
p^{*}(\tau)=1, V^{*}(\tau)=1-\frac{\tau}{\tau_{s}^{0}}, 0 \leq \tau \leq \tau_{s}=\tau_{s}^{0}
$$

i.e. parameter $\tau_{s}^{0}(2.7)$ may be treated as the dimensionless duration of braking at the constant deceleration.

The thermal behavior of a brake system, that consist of a shoe and a drum, for three specified braking actions: the impulse, unit step and trigonometric stopping actions was investigated in article (Naji and Al-Nimr, 2001). Most often for an analytical determination of average temperature in the pad/disc system three calculation schemes are used: two semi-spaces, a plane-parallel strip/the semi-space (the foundation), and two plane-parallel 
strips. The corresponding thermal problems of friction can be formulated as onedimensional boundary-value problems of heat conductivity of parabolic type. The temperature analysis for two homogeneous semi-spaces with the pressure increasing monotonically during braking, in accordance with equation (2.10), has been performed in the articles (Yevtushenko at al., 1999; Olesiak at al., 1997). The corresponding solution for two plane-parallel strips has been obtained in article (Pyryev and Yevtushenko, 2000).

\section{Statement of the problem}

In the general case, the problem of contact interaction of a pad (a plane-parallel strip) and a disc (a semi-infinite foundation) can be formulated as: the time-dependent normal pressure $p(\tau), 0 \leq \tau \leq \tau_{s}(2.1)$ in the direction of the $z$-axis of the Cartesian system of coordinates Oxyz is applied to the upper surface of the strip and to the infinity in semi-space (Fig. 1). The strip slides with the speed $V(\tau), 0 \leq \tau \leq \tau_{s}(2.4)-(2.7)$ in the direction of the $y$-axis on the surface of the semi-space. Due to friction, the heat is generated on a surface of contact $z=0$, and the elements are heated. The sum of heat fluxes, directed from a surface of contact inside each bodies, is equal to specific friction power (Barber and Comminou, 1989)

$$
q(\tau)=q_{0} q^{*}(\tau), q^{*}(\tau)=p^{*}(\tau) V^{*}(\tau), q_{0}=f V p_{0},
$$

where $f$ is a coefficient of friction, and the functions $p^{*}(\tau)$ and $V^{*}(\tau)$ have the form (2.1) and (2.4), respectively. Between contact surfaces of the strip and the foundation the heat transfer takes place with a coefficient of thermal conductivity of contact $h$. The strip surface $z=d$ is under condition of convective heat exchange with a coefficient of heat exchange $h_{s}$.

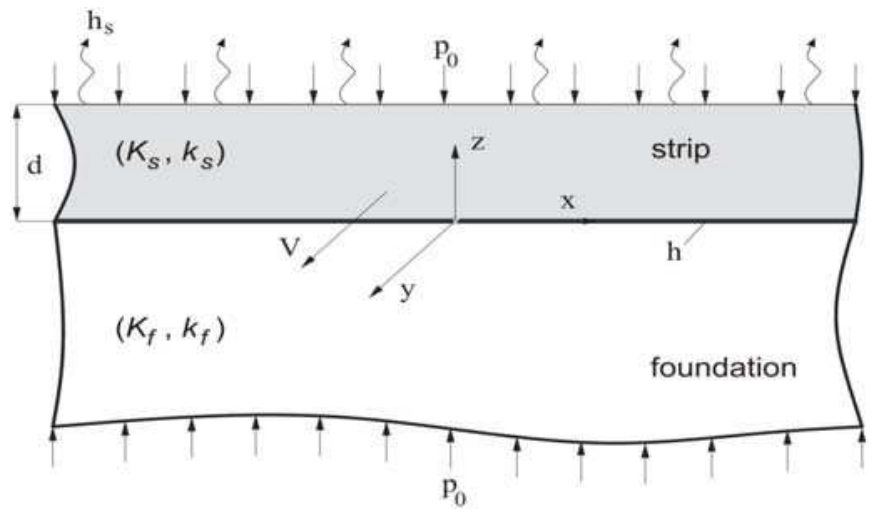

Fig. 1. Scheme of the problem

All values and the parameters concerning the strip and foundation will have bottom indexes " $s$ " and " $f$ ", respectively.

In accordance with the above-mentioned assumptions, the heat conductivity problem at friction takes form:

$$
\frac{\partial^{2} T^{*}(\zeta, \tau)}{\partial \zeta^{2}}=\frac{\partial T^{*}(\zeta, \tau)}{\partial \tau}, \quad 0<\zeta<1,0 \leq \tau \leq \tau_{s},
$$




$$
\begin{gathered}
\frac{\partial^{2} T^{*}(\zeta, \tau)}{\partial \zeta^{2}}=\frac{1}{k^{*}} \frac{\partial T^{*}(\zeta, \tau)}{\partial \tau},-\infty<\zeta<0,0 \leq \tau \leq \tau_{s^{\prime}}, \\
\left.K^{*} \frac{\partial T^{*}}{\partial \zeta}\right|_{\zeta=0-}-\left.\frac{\partial T^{*}}{\partial \zeta}\right|_{\zeta=0+}=q^{*}(\tau), \quad 0 \leq \tau \leq \tau_{s^{\prime}} \\
\left.K^{*} \frac{\partial T^{*}}{\partial \zeta}\right|_{\zeta=0-}+\left.\frac{\partial T^{*}}{\partial \zeta}\right|_{\zeta=0+}=\operatorname{Bi}\left[T^{*}(0+, \tau)-T^{*}(0-, \tau)\right], 0 \leq \tau \leq \tau_{s}, \\
\left.\frac{\partial T^{*}}{\partial \zeta}\right|_{\zeta=1}+\operatorname{Bi}_{\mathrm{s}} T^{*}(1, \tau)=0,0 \leq \tau \leq \tau_{s}, \\
T^{*}(\zeta, \tau) \rightarrow 0, \quad \zeta \rightarrow-\infty, \quad 0 \leq \tau \leq \tau_{s}, \\
T^{*}(\zeta, 0)=0, \quad-\infty<\zeta \leq 1,
\end{gathered}
$$

where

$$
\zeta=\frac{z}{d}, \quad \mathrm{Bi}_{s}=\frac{h_{s} d}{K_{s}}, K^{*}=\frac{K_{f}}{K_{s}}, \quad k^{*}=\frac{k_{f}}{k_{s}}, T_{0}=\frac{q_{0} d}{K_{s}}, T_{s, f}^{*}=\frac{T_{s, f}}{T_{0}},
$$

$K$ - coefficient of thermal conductivity; $k$ - coefficient of thermal diffusivity; $T$ - temperature; $Z$ - spatial coordinate, $q$ - has the form (3.1).

\section{Heat generation at constant friction power. Perfect contact.}

In this Chapter we consider the heat conduction problem of friction (3.2)-(3.8) of the planeparallel strip and the semi-space on the following assumptions: the elements are compressed with the constant pressure $p(\tau)(2.1)\left(p^{*}(\tau)=1\right)$, the strip slides with the constant velocity $V=V_{0}\left(V^{*}=1\right)$, and in the boundary condition (3.5) $\mathrm{Bi} \rightarrow \infty$ (perfect thermal contact i.e. $T_{f}(0, \tau)=T_{\mathrm{S}}(0, \tau)$. From the assumptions above it follows that the intensity of the frictional heat fluxes directed into each component of friction pair is equal to the specific friction power $q_{0}=f V p_{0}(3.1)\left(q^{*}(\tau)=1\right)$.

\subsection{Solution to the problem}

By applying the Laplace integral transform to the equations (3.2)-(3.8) with respect to the dimensionless time $\tau$ (Sneddon, 1972)

$$
L\left[T_{s, f}(\zeta, \tau) ; p\right] \equiv \bar{T}_{s, f}^{*}(\zeta, p)=\int_{0}^{\infty} T_{s, f}^{*}(\zeta, \tau) e^{-p \tau} d \tau
$$

we obtain

$$
\frac{d^{2} \bar{T}_{s}^{*}(\zeta, p)}{d \zeta^{2}}-p \bar{T}_{s}^{*}(\zeta, p)=0, \quad 0<\zeta<1
$$




$$
\begin{gathered}
\frac{d^{2} \bar{T}_{f}^{*}(\zeta, p)}{d \zeta^{2}}-\frac{p}{k^{*}} \bar{T}_{f}^{*}(\zeta, p)=0, \quad-\infty<\zeta<0, \\
\left.K^{*} \frac{d \bar{T}_{f}}{d \zeta}\right|_{\zeta=0-}-\left.\frac{d \bar{T}_{s}}{d \zeta}\right|_{\zeta=0+}=\frac{1}{p}, \\
\bar{T}_{f}^{*}(0, p)=\bar{T}_{s}^{*}(0, p), \\
\left.\frac{d \bar{T}_{s}^{*}(\zeta, p)}{d \zeta}\right|_{\zeta=1}+\operatorname{Bi}_{s} \bar{T}_{s}^{*}(1, p)=0, \\
\bar{T}_{f}^{*}(\zeta, p) \rightarrow 0, \quad \zeta \rightarrow-\infty .
\end{gathered}
$$

The solutions of the ordinary differential equations (4.2) and (4.3) at boundary conditions (4.4)-(4.7) have the form:

$$
\begin{gathered}
\bar{T}_{s}^{*}(\zeta, p)=\frac{\Delta_{s}(\zeta, p)}{p \sqrt{p} \Delta(p)}, \quad 0 \leq \zeta \leq 1, \\
\bar{T}_{f}^{*}(\zeta, p)=\frac{\Delta_{f}(\zeta, p)}{p \sqrt{p} \Delta(p)}, \quad-\infty<\zeta \leq 0,
\end{gathered}
$$

where

$$
\begin{gathered}
\Delta_{s}(\zeta, p)=\sqrt{p} \operatorname{ch}[(1-\zeta) \sqrt{p}]+\mathrm{Bi}_{\mathrm{s}} \operatorname{sh}[(1-\zeta) \sqrt{p}], \\
\Delta_{f}(\zeta, p)=\left[\sqrt{p} \operatorname{ch}(\sqrt{p})+\mathrm{Bi}_{\mathrm{s}} \operatorname{sh}(\sqrt{p})\right] e^{\zeta \sqrt{\frac{p}{k^{*}}},} \\
\Delta(p)=\left(\sqrt{p}+\varepsilon \mathrm{Bi}_{\mathrm{s}}\right) \operatorname{sh}(\sqrt{p})+\left(\varepsilon \sqrt{p}+\mathrm{Bi}_{\mathrm{s}}\right) \operatorname{ch}(\sqrt{p}), \\
\varepsilon=\frac{K_{f}}{K_{s}} \sqrt{\frac{k_{s}}{k_{f}}},
\end{gathered}
$$

where $\varepsilon$ is a coefficient of thermal activity of foundation's material against strip's material. Applying the inverse Laplace transform to equations (4.8)-(4.12), we obtain the dimensionless temperatures in the strip and in the foundation:

$$
T_{s, f}^{*}(\zeta, \tau)=\frac{1}{2 \pi i} \int_{\omega-i \infty}^{\omega+i \infty} \bar{T}_{s, f}^{*}(\zeta, p) e^{p \tau} d p, \tau \geq 0 .
$$

The integrals in equation (4.14) converge in semi-space Re $\mathrm{p} \equiv \omega>0$. Presence $\sqrt{p}$ in exponents of equations (4.10)-(4.12) testifies that integrands (4.8) and (4.9) in solutions (4.14) 


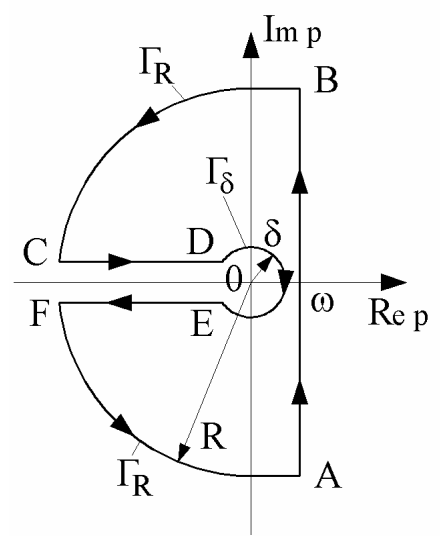

Fig. 2. Contour of integration.

and (4.15) have a branch point at the origin $p=0$. Therefore, a contour $\Gamma$ of integration in formulas (4.14) and (4.15) shall be chosen as it is shown in Fig. 2. It consists of the straight line $A B$ which is passing on distance $\omega$ parallel to imaginary axis Imp, the circles $\Gamma_{R}$ and $\Gamma_{\delta}$ with centre $O$ and radiuses $R$ and $\delta$ respectively, and boundaries $C D$ and $E F$ a cut of a complex $p$-plane along negative real axis Rep. Since the integrands (4.8) and (4.9) in solution (4.14) within the contour $\Gamma$ have no singularities, by Cauchy's theorem (Sneddon, 1972):

$$
\frac{1}{2 \pi i} \oint_{\Gamma} \bar{T}_{s, f}(\zeta, p) e^{p \tau} d p \equiv \frac{1}{2 \pi i} \int_{A B}+\frac{1}{2 \pi i} \int_{B C}+\frac{1}{2 \pi i} \int_{C D}+\frac{1}{2 \pi i} \int_{\Gamma_{\delta}}+\frac{1}{2 \pi i} \int_{E F}+\frac{1}{2 \pi i} \int_{F A}=0 .
$$

On $\operatorname{arcs} B C$ and $F A$ we have

$$
\left|\frac{\Delta_{s, f}(p)}{p \sqrt{p} \Delta(p)}\right| \leq \frac{\text { const. }}{p \sqrt{p}}
$$

and the conditions of a Jordan's lemma are carried out. Therefore, as $R \rightarrow \infty$ the corresponding integrals in equation (4.15) tend to zero and we obtain:

$$
T_{s, f}^{*}(\zeta, \tau)=T_{s, f}^{*+}(\zeta, \tau)-T_{s, f}^{*-}(\zeta, \tau)-T_{s, f}^{* 0}(\zeta, \tau),
$$

where

$$
\begin{gathered}
T_{s, f}^{*+}(\zeta, \tau)=\frac{1}{2 \pi i} \int_{C D} \bar{T}_{s, f}^{*}(\zeta, p) e^{p \tau} d p, T_{s, f}^{*-}(\zeta, \tau)=\frac{1}{2 \pi i} \int_{E F} \bar{T}_{s, f}^{*}(\zeta, p) e^{p \tau} d p, \\
T_{s, f}^{* 0}(\zeta, \tau)=\frac{1}{2 \pi i} \int_{\Gamma_{\delta}} \bar{T}_{s, f}^{*}(\zeta, p) e^{p \tau} d p .
\end{gathered}
$$

In polar coordinate system $p=\rho e^{i \varphi}$ we have $p=\rho e^{i \varphi}, \varphi=\pi, p=-\rho, \sqrt{p}=i \sqrt{\rho}$ on upper boundary $C D$ and $\varphi=-\pi, p=-\rho, \sqrt{p}=-i \sqrt{\rho}$ on bottom boundary $E F$ of a cut. Therefore, 
taking into account the equations (4.10)-(4.12) and the relations $\operatorname{ch}(i x)=\cos (x)$, $\operatorname{sh}(i x)=i \sin (x)$ (Abramovits and Stegun, 1979), we can write the integrals (4.18) in the form:

$$
T_{s, f}^{* \pm}(\zeta, \tau)= \pm \frac{1}{2 \pi i} \int_{0}^{\infty} \bar{T}_{s, f}^{* \pm}(\zeta, \rho) e^{-\rho \tau} d \rho,
$$

where

$$
\begin{gathered}
\bar{T}_{s, f}^{* \pm}(\zeta, \rho)=-\frac{\Delta_{s, f}^{ \pm}(\zeta, \rho)}{\rho \sqrt{\rho} \Delta^{ \pm}(\rho)}, \\
\Delta_{s}^{+}(\zeta, \rho)=\Delta_{s}^{-}(\zeta, \rho)=\sqrt{\rho} \cos [(1-\zeta) \sqrt{\rho}]+\mathrm{Bi}_{\mathrm{s}} \sin [(1-\zeta) \sqrt{\rho}], 0 \leq \zeta \leq 1, \\
\Delta_{f}^{ \pm}(\zeta, \rho)=\left[\sqrt{\rho} \cos (\sqrt{\rho})+\mathrm{Bi}_{\mathrm{s}} \sin (\sqrt{\rho})\right] e^{ \pm i \zeta \sqrt{\frac{\rho}{k^{*}}},-\infty<\zeta \leq 0,} \\
\Delta^{ \pm}(\rho)=\left( \pm i \varepsilon \mathrm{Bi}_{\mathrm{s}}-\sqrt{\rho}\right) \sin (\sqrt{\rho})+\left(\mathrm{Bi}_{\mathrm{s}} \pm i \varepsilon \sqrt{\rho}\right) \cos (\sqrt{\rho}) .
\end{gathered}
$$

Adding equations (4.21)-(4.24) at $x=\sqrt{\rho}$ we obtain:

$$
T_{s, f}^{*+}(\zeta, \tau)+T_{s, f}^{*-}(\zeta, \tau)=\frac{2}{\pi} \int_{0}^{\infty} F(x) G_{s, f}(\zeta, x) e^{-x^{2} \tau} d x
$$

where

$$
\begin{gathered}
F(x)=\frac{\cos (x)+\mathrm{Bi}_{\mathrm{s}} x^{-1} \sin (x)}{\left[\mathrm{Bi}_{\mathrm{s}} \cos (x)-x \sin (x)\right]^{2}+\varepsilon^{2}\left[\mathrm{Bi}_{\mathrm{s}} \sin (x)+x \cos (x)\right]^{2}}, \\
G_{s}(\zeta, x)=\varepsilon\left\{\cos [(1-\zeta) x]+\mathrm{Bi}_{\mathrm{s}} x^{-1} \sin [(1-\zeta) x]\right\}, 0 \leq \zeta \leq 1, \\
G_{f}(\zeta, x)=\varepsilon \cos \left(\zeta \frac{x}{\sqrt{k^{*}}}\right)\left[\mathrm{Bi}_{\mathrm{s}} x^{-1} \sin (x)+\cos (x)\right]- \\
-x^{-1} \sin \left(\zeta \frac{x}{\sqrt{k^{*}}}\right)\left[\mathrm{Bi}_{\mathrm{s}} \cos (x)-x \sin (x)\right],-\infty<\zeta \leq 0 .
\end{gathered}
$$

Taking the formulae (4.8)-(4.12) into account, the integrals (4.19) round the small circle $\Gamma_{\delta}$ enclosing the origin for $p=\delta e^{i \varphi},-\pi \leq \varphi \leq \pi$ and $\delta \rightarrow 0$ tends to values:

$$
T_{s}^{* 0}(\zeta, \tau)=-\frac{1+(1-\zeta) \mathrm{Bi}_{\mathrm{s}}}{\mathrm{Bi}_{\mathrm{s}}}, 0 \leq \zeta \leq 1, T_{f}^{* 0}(\zeta, \tau)=-\frac{1+\mathrm{Bi}_{\mathrm{s}}}{\mathrm{Bi}_{\mathrm{s}}},-\infty<\zeta \leq 0 .
$$

Substituting the results (4.25) and (4.29) into equations (4.17), we obtain the dimensionless temperatures in the strip and the foundation: 


$$
\begin{gathered}
T_{s}^{*}(\zeta, \tau)=\frac{1+(1-\zeta) \mathrm{Bi}_{s}}{\mathrm{Bi}_{s}}-\frac{2}{\pi} \int_{0}^{\infty} F(x) G_{s}(\zeta, x) e^{-x^{2} \tau} d x, \quad 0 \leq \zeta \leq 1, \\
T_{f}^{*}(\zeta, \tau)=\frac{1+\mathrm{Bi}_{s}}{\mathrm{Bi}_{s}}-\frac{2}{\pi} \int_{0}^{\infty} F(x) G_{f}(\zeta, x) e^{-x^{2} \tau} d x, \quad-\infty<\zeta \leq 0,
\end{gathered}
$$

where the integrands $F(x)$ and $G_{s, f}(\zeta, x)$ have the form (4.26)-(4.28).

\subsection{Two particular values of the heat transfer coefficient}

The boundary of the strip $z=d(\zeta=1)$ for $h_{s} \rightarrow \infty\left(\mathrm{Bi}_{\mathrm{s}} \rightarrow \infty\right)$ is kept at zero temperature, i.e. $T_{s}^{*}(1, \tau)=0, \tau>0$. In this case, the Laplace transformed solution of the boundary-value heat conductivity problem (3.2)-(3.8), has also the form (4.8) and (4.9), where

$$
\begin{gathered}
\Delta_{s}(\zeta, p)=\operatorname{sh}[(1-\zeta) \sqrt{p}], \quad 0 \leq \zeta \leq 1 \\
\Delta_{f}(\zeta, p)=\operatorname{sh}(\sqrt{p}) e^{\zeta \sqrt{\frac{p}{k^{*}}}}, \quad-\infty<\zeta \leq 0 \\
\Delta(p)=\frac{1}{2}(1+\varepsilon) e^{\sqrt{p}}\left(1+\lambda e^{-2 \sqrt{p}}\right) \\
-1<\lambda=\frac{1-\varepsilon}{1+\varepsilon}<1
\end{gathered}
$$

Taking the below relations into account

$$
\frac{1}{1+\lambda e^{-2 \sqrt{p}}}=\sum_{n=0}^{\infty} \Lambda^{n} e^{-2 n \sqrt{p}},
$$

where

$$
\Lambda^{n}=\left\{\begin{array}{ll}
(-1)^{n} \lambda^{n}, & 0 \leq \lambda<1 \\
|\lambda|^{n}, & -1<\lambda \leq 0
\end{array},\right.
$$

from the equation (4.34) it can be find

$$
\frac{1}{\Delta(p)}=\frac{2 e^{-\sqrt{p}}}{(1+\varepsilon)} \sum_{n=0}^{\infty} \Lambda^{n} e^{-2 n \sqrt{p}} .
$$

Substituting equations (4.32), (4.33) and (4.38) into the solutions (4.8) and (4.9), we find the transforms for temperatures in the following form:

$$
\bar{T}_{s}^{*}(\zeta, p)=\frac{1}{(1+\varepsilon)} \sum_{n=0}^{\infty} \Lambda^{n} \bar{T}_{s, n}^{*}(\zeta, p), \quad 0 \leq \zeta \leq 1,
$$




$$
\begin{gathered}
\bar{T}_{s, n}^{*}(\zeta, p)=\frac{1}{p \sqrt{p}}\left[e^{-(2 n+\zeta) \sqrt{p}}-e^{-(2 n+2-\zeta) \sqrt{p}}\right], n=0,1,2, \ldots, \\
\bar{T}_{f}^{*}(\zeta, p)=\frac{1}{(1+\varepsilon)} \sum_{n=0}^{\infty} \Lambda^{n} \bar{T}_{f, n}^{*}(\zeta, p), \quad-\infty<\zeta \leq 0, \\
\bar{T}_{f, n}^{*}(\zeta, p)=\frac{1}{p \sqrt{p}}\left[e^{-\left(2 n-\frac{\zeta}{\sqrt{k^{*}}}\right) \sqrt{p}}-e^{-\left(2 n+2-\frac{\zeta}{\sqrt{k^{*}}}\right) \sqrt{p}}\right], n=0,1,2, \ldots .
\end{gathered}
$$

Applying the inversion formula (Bateman and Erdelyi, 1954)

$$
L^{-1}\left[\frac{e^{-a \sqrt{p}}}{p \sqrt{p}} ; \tau\right]=2 \sqrt{\tau} \operatorname{ierfc}\left(\frac{a}{2 \sqrt{\tau}}\right), a>0,
$$

to equation (4.39)-(4.42), we obtain the temperatures in the strip and in the foundation:

$$
\begin{gathered}
T_{s}^{*}(\zeta, \tau)=\frac{1}{(1+\varepsilon)} \sum_{n=0}^{\infty} \Lambda^{n} T_{s, n}^{*}(\zeta, \tau), \quad 0 \leq \zeta \leq 1, \tau \geq 0, \\
T_{s, n}^{*}(\zeta, \tau)=2 \sqrt{\tau}\left[\operatorname{ierfc}\left(\frac{2 n+\zeta}{2 \sqrt{\tau}}\right)-\operatorname{ierfc}\left(\frac{2 n+2-\zeta}{2 \sqrt{\tau}}\right)\right], n=0,1,2, \ldots, \\
T_{f}^{*}(\zeta, \tau)=\frac{1}{(1+\varepsilon)} \sum_{n=0}^{\infty} \Lambda^{n} T_{f, n}^{*}(\zeta, \tau), \quad-\infty<\zeta \leq 0, \quad \tau \geq 0, \\
T_{f, n}^{*}(\zeta, \tau)=2 \sqrt{\tau}\left[\operatorname{ierfc}\left(\frac{2 n \sqrt{k^{*}}-\zeta}{2 \sqrt{k^{*} \tau}}\right)-\operatorname{ierfc}\left(\frac{(2 n+2) \sqrt{k^{*}}-\zeta}{2 \sqrt{k^{*} \tau}}\right)\right], n=0,1,2, \ldots
\end{gathered}
$$

From formulas (4.44)-(4.47) it can be easily proved, that boundary and initial conditions (3.4)-(3.8), at $\mathrm{Bi}_{\mathrm{s}} \rightarrow \infty$ are satisfied.

At $h_{\mathrm{s}} \rightarrow 0\left(\mathrm{Bi}_{\mathrm{s}} \rightarrow 0\right)$ the surface of the strip $\zeta=1$ is insulated:

$$
\left.\frac{\partial T_{s}^{*}}{\partial \zeta}\right|_{\zeta=1}=0, \quad \tau>0 .
$$

The solution of the heat conduction equations (3.2) and (3.3), satisfying boundary and initial conditions (3.4)-(3.8) and (4.48), also can be found in the form of (4.44) and (4.46), when

$$
\begin{gathered}
T_{s, n}^{*}(\zeta, \tau)=2 \sqrt{\tau}\left[\operatorname{ierfc}\left(\frac{2 n+\zeta}{2 \sqrt{\tau}}\right)+\operatorname{ierfc}\left(\frac{2 n+2-\zeta}{2 \sqrt{\tau}}\right)\right], n=0,1,2, \ldots, \\
T_{f, n}^{*}(\zeta, \tau)=2 \sqrt{\tau}\left[\operatorname{ierfc}\left(\frac{2 n \sqrt{k^{*}}-\zeta}{2 \sqrt{k^{*} \tau}}\right)+\operatorname{ierfc}\left(\frac{(2 n+2) \sqrt{k^{*}}-\zeta}{2 \sqrt{k^{*} \tau}}\right)\right], n=0,1,2, \ldots
\end{gathered}
$$


If the properties of materials of the strip and the foundation are the same, then from formulae (4.13), (4.25) and (4.37), that $\varepsilon=1, \lambda=0, \Lambda=0$. Hence, for $n=0$ from solutions (4.44)(4.47), (4.50) and (4.50) are obtained

$$
\begin{aligned}
& T_{s}^{*}(\zeta, \tau)=\sqrt{\tau}\left[\operatorname{ierfc}\left(\frac{\zeta}{2 \sqrt{\tau}}\right) \mp \operatorname{ierfc}\left(\frac{2-\zeta}{2 \sqrt{\tau}}\right)\right], 0 \leq \zeta \leq 1, \tau \geq 0, \\
& T_{f}^{*}(\zeta, \tau)=\sqrt{\tau}\left[\operatorname{ierfc}\left(\frac{-\zeta}{2 \sqrt{\tau}}\right) \mp \operatorname{ierfc}\left(\frac{2-\zeta}{2 \sqrt{\tau}}\right)\right],-\infty<\zeta \leq 0, \quad \tau \geq 0,
\end{aligned}
$$

where the upper sign should be taken when the surface of the strip $z=d(\zeta=1)$ is kept at zero temperature, and bottom - when this surface is insulated.

Finally, we note that the solution of the corresponding thermal problem of friction for two homogeneous semi-spaces was found in the monograph (Grylytskyy, 1996)

$$
T^{*}(\zeta, \tau)=\frac{2 \sqrt{\tau}}{(1+\varepsilon)} \begin{cases}\operatorname{ierfc}\left(\frac{\zeta}{2 \sqrt{\tau}}\right), & 0 \leq \zeta<\infty, \tau \geq 0, \\ \operatorname{ierfc}\left(\frac{-\zeta}{2 \sqrt{k^{*} \tau}}\right), & -\infty<\zeta \leq 0, \tau \geq 0\end{cases}
$$

The distribution of dimensionless temperature in the semi-space, which is heated up on a surface $\zeta=0$ with a uniform heat flux of intensity $q_{0}$ has the well-known form (Carslaw and Jaeger, 1959):

$$
T^{*}(\zeta, \tau)=2 \sqrt{\tau} \operatorname{ierfc}\left(\frac{\zeta}{2 \sqrt{\tau}}\right), \quad 0 \leq \zeta<\infty, \tau \geq 0
$$

\section{Heat generation at constant friction power. Imperfect contact.}

In this Chapter the impact of thermal resistance on the contact surface on the temperature distribution in strip-foundation system is investigated. For this purpose, we consider the heat conduction problem of friction (3.2)-(3.8) on the following assumptions: constant pressure $p(\tau)(2.1)\left(p^{*}(\tau)=1\right)$, constant velocity $V=V_{0}\left(V^{*}=1\right)$ and zero temperature on the upper surface of the strip, i.e. in the boundary condition (3.6) $\mathrm{Bi}_{\mathrm{s}} \rightarrow \infty$.

\subsection{Solution to the problem}

Solution of a boundary-value problem of heat conduction in friction (3.2)-(3.8) by applying the Laplace integral transforms (4.1) has form

$$
\bar{T}_{s, f}^{*}(\zeta, p)=\frac{\Delta_{s, f}(\zeta, p)}{p \Delta(p)},
$$

where

$$
\Delta_{s}(\zeta, p)=\left(\varepsilon+\frac{\mathrm{Bi}}{\sqrt{p}}\right) \operatorname{sh}[(1-\zeta) \sqrt{p}], \quad 0 \leq \zeta \leq 1
$$




$$
\begin{gathered}
\Delta_{f}(\zeta, p)=\left[\operatorname{ch} \sqrt{\mathrm{p}}+\frac{\mathrm{Bi}}{\sqrt{p}} \operatorname{sh} \sqrt{p}\right] e^{\zeta \sqrt{\frac{p}{k^{*}}}},-\infty<\zeta \leq 0, \\
\Delta(p)=\varepsilon \operatorname{Bi~} \operatorname{sh} \sqrt{p}+(2 \varepsilon \sqrt{p}+\mathrm{Bi}) \operatorname{ch} \sqrt{p} .
\end{gathered}
$$

Applying the inverse Laplace transform to Eqs. (5.1)-(5.4) with integration along the same contour as in Fig. 2, we obtain the dimensionless temperatures in the strip and in the foundation:

$$
T_{s, f}^{*}(\zeta, \tau)=T_{s, f}^{* 0}(\zeta)-\frac{2}{\pi} \int_{0}^{\infty} F(x) G_{s}(\zeta, x) e^{-x^{2} \tau} d x, \tau \geq 0
$$

where

$$
\begin{gathered}
T_{s}^{* 0}(\zeta)=1-\zeta, 0 \leq \zeta \leq 1, T_{f}^{* 0}(\zeta)=\frac{1+\mathrm{Bi}}{\mathrm{Bi}},-\infty<\zeta \leq 0, \\
F(x)=\frac{\cos x+\mathrm{Bi} x^{-1} \sin x}{(\mathrm{Bi} \cos x)^{2}+\varepsilon^{2}(\mathrm{Bi} \sin x+2 x \cos x)^{2}}, \\
G_{s}(\zeta, x)=\varepsilon \operatorname{Bi} x^{-1} \sin [(1-\zeta) x], 0 \leq \zeta \leq 1, \\
G_{f}(\zeta, x)=\varepsilon\left(\frac{\mathrm{Bi}}{x} \sin x+2 \cos x\right) \cos \left(\zeta x / \sqrt{k^{*}}\right)-\frac{\mathrm{Bi}}{x} \cos x \sin \left(\zeta x / \sqrt{k^{*}}\right),-\infty<\zeta \leq 0 .
\end{gathered}
$$

The maximum temperature is reached on the friction surface $\zeta=0$. In order to determine the maximum temperature, we use the solutions (5.5) at $T_{s}^{* 0}(\zeta)=1$ and the integrands (5.7) as well as

$$
G_{s}(0, x)=\varepsilon \operatorname{Bi} x^{-1} \sin x, G_{f}(0, x)=\varepsilon\left(\operatorname{Bi} x^{-1} \sin x+2 \cos x\right) .
$$

Let us define the heat flux intensities in the strip and in semi-space as following:

$$
q_{s}(z, t) \equiv-K_{s} \frac{\partial T_{s}(z, t)}{\partial z}, 0 \leq z \leq d, t \geq 0, q_{f}(z, t) \equiv K_{f} \frac{\partial T_{f}(z, t)}{\partial z},-\infty<z \leq 0, t \geq 0,
$$

or with taking (3.9) under consideration in the dimensionless form:

$$
\begin{gathered}
q_{s}^{*}(\xi, \tau) \equiv \frac{q_{s}(z, t)}{q}=-\frac{\partial T_{s}^{*}(\zeta, \tau)}{\partial \zeta}, 0 \leq \zeta \leq 1, \quad \tau \geq 0, \\
q_{f}^{*}(\zeta, \tau) \equiv \frac{q_{f}(z, t)}{q}=K^{*} \frac{\partial T_{f}^{*}(\zeta, \tau)}{\partial \zeta},-\infty<\zeta \leq 0, \tau \geq 0 .
\end{gathered}
$$

With taking solutions for dimensionless temperatures (5.5)-(5.9) under consideration, from the formulae (5.12) and (5.13) we found: 


$$
\begin{gathered}
q_{s}^{*}(\zeta, \tau)=1-\frac{2 \varepsilon}{\pi} \int_{0}^{\infty} F(x) Q_{s}(\zeta, x) e^{-x^{2} \tau} d x, \quad 0 \leq \zeta \leq 1, \tau \geq 0, \\
q_{f}^{*}(\zeta, \tau)=\frac{2 \varepsilon}{\pi} \int_{0}^{\infty} F(x) Q_{f}(\zeta, x) e^{-x^{2} \tau} d x, \quad-\infty<\zeta \leq 0, \tau \geq 0, \\
Q_{s}(\zeta, x)=\operatorname{Bi} \cos [(1-\zeta) x], 0 \leq \zeta \leq 1, \\
Q_{f}(\zeta, x)=\varepsilon(\operatorname{Bisin} x+2 x \cos x) \sin \left(\zeta x / \sqrt{k^{*}}\right)+\operatorname{Bi} \cos x \cos \left(\zeta x / \sqrt{k^{*}}\right),-\infty<\zeta \leq 0 .
\end{gathered}
$$

On the friction surface $\zeta=0$ from the formulae (5.16) and (5.17) leads $Q_{f}(0, x)=Q_{s}(0, x)=\operatorname{Bicos} x$ and from (5.14), (5.15) we found $q_{f}^{*}(0, \tau)+q_{s}^{*}(0, \tau)=1, \tau \geq 0$, which means that boundary condition (3.4) is satisfied $\left(q^{*}(\tau)=1\right)$. Spikes of temperature and heat flux intensities both on the contact surface $\zeta=0$ we found from solutions of (5.5)(5.9) and (5.14)-(5.17) in the form:

$$
\begin{aligned}
& T_{s}^{*}(0, \tau)-T_{f}^{*}(0, \tau)=-\frac{1}{\mathrm{Bi}}+\frac{4 \varepsilon}{\pi} \int_{0}^{\infty} F(x) e^{-x^{2} \tau} \cos x d x, \tau \geq 0, \\
& q_{f}^{*}(0, \tau)-q_{s}^{*}(0, \tau)=-1+\frac{4 \varepsilon \mathrm{Bi}}{\pi} \int_{0}^{\infty} F(x) e^{-x^{2} \tau} \cos x d x, \tau \geq 0,
\end{aligned}
$$

whence follows, that the boundary condition (3.5) is satisfied.

Dimensionless temperatures and heat flux intensities in case of perfect contact between strip and foundation $(h \rightarrow \infty$ or $\mathrm{Bi} \rightarrow \infty)$ can be found from the Eqs. (5.5), (5.14) and (5.15) at $T_{f}^{* 0}(\zeta)=1$ and the integrands in the forms:

$$
\begin{gathered}
F(x)=\frac{x^{-1} \sin (x)}{\cos ^{2}(x)+\varepsilon^{2} \sin ^{2}(x)}, \\
G_{s}(\zeta, x)=\varepsilon x^{-1} \sin [(1-\zeta) x], Q_{s}(\zeta, x)=\cos [(1-\zeta) x], 0 \leq \zeta \leq 1, \\
G_{f}(\zeta, x)=\varepsilon x^{-1} \sin (x) \cos \left(\zeta x / \sqrt{k^{*}}\right)-x^{-1} \cos (x) \sin \left(\zeta x / \sqrt{k^{*}}\right),-\infty<\zeta \leq 0, \\
Q_{f}(\zeta, x)=\varepsilon \sin (x) \sin \left(\zeta x / \sqrt{k^{*}}\right)+\cos (x) \cos \left(\zeta x / \sqrt{k^{*}}\right),-\infty<\zeta \leq 0 .
\end{gathered}
$$

On the contact surface $\zeta=0$ from Eqs. (5.20)-(5.23) result as following

$$
G_{s}(0, x)=G_{f}(0, x)=\varepsilon x^{-1} \sin x, Q_{s}(0, x)=Q_{f}(0, x)=\cos x .
$$

The formulae (5.20)-(5.24) from the solution of the contact problem with heat generation due to friction at perfect thermal contact between strip and foundation, were obtained in Chapter four. 


\subsection{Asymptotic solutions}

For large values of the parameter $p$ of Laplace integral transform (4.1) the solutions (5.1)(5.4) will take form:

$$
\begin{aligned}
& \bar{T}_{s}^{*}(\zeta, p) \cong \frac{(\varepsilon+\mathrm{Bi} / \sqrt{p})}{2 \varepsilon p(\alpha+\sqrt{p})} e^{-\zeta \sqrt{p}}, \bar{q}_{s}^{*}(\zeta, p) \cong \frac{(\varepsilon \sqrt{p}+\mathrm{Bi})}{2 \varepsilon p(\alpha+\sqrt{p})} e^{-\zeta \sqrt{p}}, 0 \leq \zeta \leq 1, \\
& \bar{T}_{f}^{*}(\zeta, p) \cong \frac{(1+\mathrm{Bi} / \sqrt{p})}{2 \varepsilon p(\alpha+\sqrt{p})} e^{\zeta \sqrt{\frac{p}{k^{*}}}}, \bar{q}_{f}^{*}(\zeta, p) \cong \frac{(\sqrt{p}+\mathrm{Bi})}{2 p(\alpha+\sqrt{p})} e^{\zeta \sqrt{\frac{p}{k^{*}}}},-\infty<\zeta \leq 0,
\end{aligned}
$$

where

$$
\alpha=\frac{(1+\varepsilon)}{2 \varepsilon} \mathrm{Bi} .
$$

By using the relations (Bateman and Erdelyi, 1954)

$$
\begin{gathered}
L^{-1}\left[\frac{e^{-|\zeta| \sqrt{p}}}{\sqrt{p}(\alpha+\sqrt{p})} ; \tau\right]=e^{\alpha|\zeta|+\alpha^{2} \tau} \operatorname{erfc}\left(\frac{|\zeta|}{2 \sqrt{\tau}}+\alpha \sqrt{\tau}\right), \\
L^{-1}\left[\frac{\alpha e^{-|\zeta| \sqrt{p}}}{p(\alpha+\sqrt{p})} ; \tau\right]=\operatorname{erfc}\left(\frac{|\zeta|}{2 \sqrt{\tau}}\right)-e^{\alpha|\zeta|+\alpha^{2} \tau} \operatorname{erfc}\left(\frac{|\zeta|}{2 \sqrt{\tau}}+\alpha \sqrt{\tau}\right), \\
L^{-1}\left[\frac{e^{-|\zeta| \sqrt{p}}}{p \sqrt{p}(\alpha+\sqrt{p})} ; \tau\right]= \\
=\frac{2}{\alpha} \sqrt{\frac{\tau}{\pi}} e^{-\frac{\zeta^{2}}{4 \tau}}-\left(\frac{|\zeta|}{\alpha}+\frac{1}{\alpha^{2}}\right) \operatorname{erfc}\left(\frac{|\zeta|}{2 \sqrt{\tau}}\right)+\frac{1}{\alpha^{2}} e^{\alpha|\zeta|+\alpha^{2} \tau} \operatorname{erfc}\left(\frac{|\zeta|}{2 \sqrt{\tau}}+\alpha \sqrt{\tau}\right),
\end{gathered}
$$

we have obtained from Eqs. (5.25), (5.26) the asymptotic formulae for dimensionless temperature and heat flux intensities both for the strip and foundation at small values of the dimensionless time $0 \leq \tau<<1$ :

$$
\begin{gathered}
T_{s}^{*}(\zeta, \tau) \cong \frac{2 \sqrt{\tau}}{(1+\varepsilon)} \operatorname{ierfc}\left(\frac{\zeta}{2 \sqrt{\tau}}\right)-\frac{\lambda}{2 \alpha}\left[\operatorname{erfc}\left(\frac{\zeta}{2 \sqrt{\tau}}\right)-e^{\alpha|\zeta|+\alpha^{2} \tau} \operatorname{erfc}\left(\frac{\zeta}{2 \sqrt{\tau}}+\alpha \sqrt{\tau}\right)\right], 0 \leq \zeta \leq 1, \\
T_{f}^{*}(\zeta, \tau) \cong \frac{2 \sqrt{\tau}}{(1+\varepsilon)} \operatorname{ierfc}\left(\frac{|\zeta|}{2 \sqrt{k^{*} \tau}}\right)+\frac{\lambda}{2 \alpha \varepsilon}\left[\operatorname{erfc}\left(\frac{|\zeta|}{2 \sqrt{k^{*} \tau}}\right)-e^{\alpha \frac{|\zeta|}{\sqrt{k^{*}}}+\alpha^{2} \tau} \operatorname{erfc}\left(\frac{|\zeta|}{2 \sqrt{k^{*} \tau}}+\alpha \sqrt{\tau}\right)\right], \\
-\infty<\zeta \leq 0 \\
q_{s}^{*}(\zeta, \tau) \cong \frac{1}{(1+\varepsilon)} \operatorname{erfc}\left(\frac{\zeta}{2 \sqrt{\tau}}\right)-\frac{\lambda}{2} e^{\alpha|\zeta|+\alpha^{2} \tau} \operatorname{erfc}\left(\frac{\zeta}{2 \sqrt{\tau}}+\alpha \sqrt{\tau}\right), 0 \leq \zeta \leq 1,
\end{gathered}
$$




$$
q_{f}^{*}(\zeta, \tau) \cong \frac{\varepsilon}{(1+\varepsilon)} \operatorname{erfc}\left(\frac{|\zeta|}{2 \sqrt{k^{*} \tau}}\right)+\frac{\lambda}{2} e^{\alpha \frac{|\zeta|}{\sqrt{k^{*}}+\alpha^{2} \tau}} \operatorname{erfc}\left(\frac{|\zeta|}{2 \sqrt{k^{*} \tau}}+\alpha \sqrt{\tau}\right),-\infty<\zeta \leq 0,
$$

where

$$
\lambda=\frac{1-\varepsilon}{1+\varepsilon} .
$$

The dimensionless temperatures (5.31) and (5.32) tends to zero as $\tau \rightarrow 0$, which means that initial conditions (3.8) are satisfied.

On the contact surface of the strip and foundation $\zeta=0$ we find from the solutions of (5.31)-(5.34) that:

$$
\begin{gathered}
T_{s}^{*}(0, \tau) \cong \frac{2}{(1+\varepsilon)} \sqrt{\frac{\tau}{\pi}}-\frac{\lambda}{2 \alpha}\left[1-e^{\alpha^{2} \tau} \operatorname{erfc}(\alpha \sqrt{\tau})\right], \quad 0 \leq \tau<<1, \\
T_{f}^{*}(0, \tau) \cong \frac{2}{(1+\varepsilon)} \sqrt{\frac{\tau}{\pi}}+\frac{\lambda}{2 \alpha \varepsilon}\left[1-e^{\alpha^{2} \tau} \operatorname{erfc}(\alpha \sqrt{\tau})\right], \\
q_{s}^{*}(0, \tau) \cong \frac{1}{(1+\varepsilon)}-\frac{\lambda}{2} e^{\alpha^{2} \tau} \operatorname{erfc}(\alpha \sqrt{\tau}), q_{f}^{*}(0, \tau) \cong \frac{\varepsilon}{(1+\varepsilon)}+\frac{\lambda}{2} e^{\alpha^{2} \tau} \operatorname{erfc}(\alpha \sqrt{\tau}), 0<\tau<<1 .
\end{gathered}
$$

By taking (5.27) and (5.35) into account, from the Eqs. (5.36) and (5.37) we find:

$$
\begin{gathered}
T_{s}^{*}(0, \tau)-T_{f}^{*}(0, \tau)=-\frac{\lambda}{\mathrm{Bi}}\left[1-e^{\alpha^{2} \tau} \operatorname{erfc}(\alpha \sqrt{\tau})\right], 0 \leq \tau<<1, \\
q_{f}^{*}(0, \tau)+q_{s}^{*}(0, \tau)=1, q_{f}^{*}(0, \tau)-q_{s}^{*}(0, \tau)=-\lambda\left[1-e^{\alpha^{2} \tau} \operatorname{erfc}(\alpha \sqrt{\tau})\right], 0<\tau<<1,
\end{gathered}
$$

which also means that received asymptotic solution satisfies the boundary conditions (3.4) (where $q^{*}(\tau)=1$ ) and (3.5).

As results from solutions (5.31) and (5.32), at small Fourier number values $\tau$ the temperature of strip and foundation in case of perfect thermal contact $(\mathrm{Bi} \rightarrow \infty)$, can be found with use of solution of the friction heat for two semi-spaces (Yevtushenko and Kuciej, 2009a)

$$
\begin{aligned}
& T_{s}^{*}(\zeta, \tau) \cong \frac{2 \sqrt{\tau}}{(1+\varepsilon)} \operatorname{ierfc}\left(\frac{\zeta}{2 \sqrt{\tau}}\right), 0 \leq \zeta<\infty, \\
& T_{f}^{*}(\zeta, \tau) \cong \frac{2 \sqrt{\tau}}{(1+\varepsilon)} \operatorname{ierfc}\left(-\frac{\zeta}{2 \sqrt{k^{*} \tau}}\right), \quad-\infty<\zeta \leq 0, \quad 0 \leq \tau<<1 .
\end{aligned}
$$

At small values of the parameter $p$ from solutions (5.1)-(5.4) we obtain:

$$
\bar{T}_{s}^{*}(\zeta, p) \cong \frac{(1-\zeta)}{(2+\mathrm{Bi})}\left[\frac{(2+\mathrm{Bi}) \beta+\sqrt{p}}{p(\beta+\sqrt{p})}\right], \bar{q}_{f}^{*}(\zeta, p) \cong \frac{(\varepsilon \sqrt{p}+\mathrm{Bi})}{2 \varepsilon p(2+\mathrm{Bi})(\alpha+\sqrt{p})}, 0 \leq \zeta \leq 1,
$$




$$
\begin{aligned}
\bar{T}_{f}^{*}(\zeta, p) \cong \frac{(1+\mathrm{Bi}) \beta}{\operatorname{Bi}}\left[\frac{1+\zeta \sqrt{p / k^{*}}}{p(\beta+\sqrt{p})}\right], \bar{q}_{f}^{*}(\zeta, p) \cong \frac{(1+\mathrm{Bi})}{(2+\mathrm{Bi}) \sqrt{p}(\alpha+\sqrt{p})}\left(1+\zeta \sqrt{\frac{p}{k^{*}}}\right), \\
-\infty<\zeta \leq 0,
\end{aligned}
$$

where

$$
\beta=\frac{\mathrm{Bi}}{\varepsilon(2+\mathrm{Bi})} .
$$

By applying the Laplace inversion formulae (4.43) we obtain from Eqs. (5.41), (5.42) dimensionless temperatures and heat flux intensities in the strip and in the foundation at large values $(\tau>>1)$ of the dimensionless time $\tau$ :

$$
\begin{gathered}
T_{s}^{*}(\zeta, \tau) \cong(1-\zeta)\left[1-\frac{(1+\mathrm{Bi})}{(2+\mathrm{Bi})} e^{\beta^{2} \tau} \operatorname{erfc}(\beta \sqrt{\tau})\right], 0 \leq \zeta \leq 1, \\
T_{f}^{*}(\zeta, \tau) \cong \frac{(1+\mathrm{Bi})}{\mathrm{Bi}}\left[1-\left(1-\beta \frac{\zeta}{\sqrt{k^{*}}}\right) e^{\beta^{2} \tau} \operatorname{erfc}(\beta \sqrt{\tau})\right],-\infty<\zeta \leq 0, \\
q_{s}^{*}(\zeta, \tau) \cong 1-\frac{(1+\mathrm{Bi})}{(2+\mathrm{Bi})} e^{\beta^{2} \tau} \operatorname{erfc}(\beta \sqrt{\tau}), 0 \leq \zeta \leq 1, \\
q_{f}^{*}(\zeta, \tau) \cong \frac{(1+\mathrm{Bi})}{(2+\mathrm{Bi})}\left[\frac{\zeta}{\sqrt{\pi k^{*} \tau}}+\left(1-\beta \frac{\zeta}{\sqrt{k^{*}}}\right) e^{\beta^{2} \tau} \operatorname{erfc}(\beta \sqrt{\tau})\right],-\infty<\zeta \leq 0 .
\end{gathered}
$$

From the formulae (5.44)-(5.47) the temperatures and heat flux intensities on the contact surface are found in the form:

$$
\begin{gathered}
T_{s}^{*}(0, \tau) \cong 1-\frac{(1+\mathrm{Bi})}{(2+\mathrm{Bi})} e^{\beta^{2} \tau} \operatorname{erfc}(\beta \sqrt{\tau}), T_{f}^{*}(0, \tau) \cong \frac{(1+\mathrm{Bi})}{\mathrm{Bi}}\left[1-e^{\beta^{2} \tau} \operatorname{erfc}(\beta \sqrt{\tau})\right], \tau>>1 \\
q_{s}^{*}(0, \tau) \cong 1-\frac{(1+\mathrm{Bi})}{(2+\mathrm{Bi})} e^{\beta^{2} \tau} \operatorname{erfc}(\beta \sqrt{\tau}), q_{f}^{*}(0, \tau) \cong \frac{(1+\mathrm{Bi})}{(2+\mathrm{Bi})} e^{\beta^{2} \tau} \operatorname{erfc}(\beta \sqrt{\tau}), \tau>>1
\end{gathered}
$$

From the formulae (5.48) and (5.49), is easy to find that boundary conditions (3.4) (where $\left.q^{*}(\tau)=1\right)$ and (3.5) are satisfied.

In addition, from (5.46) and (5.47) follows, that at fixed enough big value of Fourier number $\tau$, the heat flux is constant along strip thickness and in foundation its value decreases linearly with distance from contact surface.

The dimensionless temperatures in the strip and in the foundation with assumption of theirs perfect thermal contact ( $\mathrm{Bi} \rightarrow \infty$ ) can be found from solutions (5.44) and (5.45) in the form: 


$$
\begin{gathered}
T_{s}^{*}(\zeta, \tau) \cong(1-\zeta)\left[1-e^{\left(\frac{\sqrt{\tau}}{\varepsilon}\right)^{2}} \operatorname{erfc}\left(\frac{\sqrt{\tau}}{\varepsilon}\right)\right], 0 \leq \zeta \leq 1, \tau>1, \\
T_{f}^{*}(\zeta, \tau) \cong 1-\left(1-\frac{\zeta}{\varepsilon \sqrt{k^{*}}}\right) e^{\left(\frac{\sqrt{\tau}}{\varepsilon}\right)^{2}} \operatorname{erfc}\left(\frac{\sqrt{\tau}}{\varepsilon}\right),-\infty<\zeta \leq 0, \tau>1.1-e^{\alpha^{2} \tau} \operatorname{erfc}(\alpha \sqrt{\tau}) .
\end{gathered}
$$

Setting in the above equations $\zeta=0$, we received the equality of strip and foundation temperatures on the contact surface:

$$
T_{s}^{*}(0, \tau)=T_{f}^{*}(0, \tau) \cong 1-e^{\left(\frac{\sqrt{\tau}}{\varepsilon}\right)^{2}} \operatorname{erfc}\left(\frac{\sqrt{\tau}}{\varepsilon}\right), \tau>>1 .
$$

\section{Heat generation of braking with constant deceleration}

In this Chapter we investigate the influence of the thermal resistance on the contact surface, and of the convective cooling on the upper surface of the strip (pad), with the constant pressure $\left(p^{*}(\tau)=1\right)$ and linear decreasing speed of sliding (breaking with constant deceleration) (2.10) taken into account. To solve a boundary problem of heat conductivity, we shall use the solutions achieved in Chapters four and five in case of constant power of friction $\left(q^{*}(\tau)=1, \tau \geq 0\right)$.

The corresponding solution to a case of braking with constant deceleration (2.10) is received by Duhamel's theorem in the form of (Luikov, 1968):

$$
\hat{T}^{*}(\zeta, \tau)=\int_{0}^{\tau} q^{*}(s) \frac{\partial}{\partial s} T^{*}(\zeta, \tau-s) d s,-\infty<\zeta \leq 1,0 \leq \tau \leq \tau_{s} .
$$

Substituting the dimensionless intensity of a heat flux $q^{*}(\tau)(3.1),(2.10)$ and the temperature obtained $T^{*}(\zeta, \tau)$ in the fourth Chapter (4.30), (4.31) to the right parts of formulae (6.1), after integration we obtain a formulae for braking with constant deceleration in case of the perfect thermal contact (between the strip and foundation), and the convective cooling on the upper surface of the strip:

$$
\hat{T}^{*}(\zeta, \tau)=\frac{2}{\pi} \int_{0}^{\infty} F(x) G(\zeta, x) P(\tau, x) d x, \quad-\infty<\zeta \leq 1,0 \leq \tau \leq \tau_{s},
$$

where

$$
P(\tau, x)=1-\frac{\tau}{\tau_{s}}-e^{-x^{2} \tau}+\frac{1-e^{-x^{2} \tau}}{\tau_{s} x^{2}}, P(\tau, 0)=0,
$$

functions $F(x)$ and $G(x)$ has the form (4.26)-(4.28) accordingly. 
To determine the solution to a case of braking with constant deceleration when the thermal resistance occurs on a surface of contact $(\mathrm{Bi} \geq 0)$, and the zero temperature on the upper surface of the strip is maintained $\left(\mathrm{Bi}_{\mathrm{s}} \rightarrow \infty\right)$, we have used the solutions obtained in Chapter five (5.5). For this case we obtain the solution in the form of (6.2), where functions $F(x)$ and $G(x)$ have the form (5.20)-(5.22) and function $P(\tau, x)$ has the form (6.3).

\section{Heat generation of braking with the time-dependent and fluctuations of the pressure}

In this Chapter we consider the general case of braking (3.2)-(3.8), having taken into account the time-dependent normal pressure $p(\tau)$ (2.1), the velocity $V(\tau), 0 \leq \tau \leq \tau_{s}$ (2.4)-(2.8) and the boundary condition of the zero temperature on the upper surface of the strip i.e. $\mathrm{Bi}_{\mathrm{s}} \rightarrow \infty$ (3.6).

The solution $T^{*}(\zeta, \tau)$ to a boundary-value problem of heat conductivity (3.2)-(3.8) in the case when the bodies are compressed with constant pressure $p_{0}$, and the strip is sliding with a constant speed $V_{0}$ on a surface of foundation $\left(q^{*}(\tau)=1, \tau \geq 0\right)$, has been obtained in Chapter six in the form (5.5)-(5.9).

Substituting the temperature $T^{*}(\zeta, \tau)(5.5)$ to the right part of equation (6.1) and changing the order of the integration, we obtain

$$
\hat{T}^{*}(\zeta, \tau)=\frac{2}{\pi} \int_{0}^{\infty} F(x) G(\zeta, x) P(\tau, x) d x, \quad-\infty<\zeta \leq 1,0 \leq \tau \leq \tau_{s},
$$

where

$$
P(\tau, x)=x^{2} \int_{0}^{\tau} q^{*}(s) e^{-x^{2}(\tau-s)} d s, 0 \leq x<\infty, 0 \leq \tau \leq \tau_{s}
$$

functions $F(x)$ and $G(\zeta, x)$ take the form (5.7) and (5.8), accordingly. Taking the form of the dimensionless intensity of a heat flux $q^{*}(\tau)$ (3.1) into account, the function $P(\tau, x)$ (7.2) can be written as

$$
P(\tau, x)=P_{1}(\tau, x)-\frac{a}{\tau_{s}^{0}} P_{2}(\tau, x)
$$

where

$$
P_{i}(\tau, x)=x^{2} \int_{0}^{\tau} p^{*}(s) V_{i}^{*}(s) e^{-x^{2}(\tau-s)} d s, 0 \leq x<\infty, 0 \leq \tau \leq \tau_{s}, i=1,2
$$

Substituting in equation (7.4) the functions $p^{*}(\tau)(2.1)$ and $V_{i}^{*}(s), i=1,2$ (2.4), (2.5), after integration we find

$$
P_{i}(\tau, x)=Q_{i}(\tau, x)+a R_{i}(\tau, x), 0 \leq x<\infty, 0 \leq \tau \leq \tau_{s}, i=1,2,
$$


where

$$
\begin{aligned}
& Q_{1}(\tau, x)=\left(1+\frac{1}{\tau_{s}^{0} \alpha_{m}^{2}}\right)\left[J_{0}(\tau, x, 0)-J_{0}\left(\tau, x, \alpha_{m}\right)\right]-\frac{1}{\tau_{s}^{0}}\left[J_{1}(\tau, x, 0)-J_{1}\left(\tau, x, \alpha_{m}\right)\right]- \\
& \quad-\frac{1}{\tau_{s}^{0} \alpha_{m}^{2}}\left[J_{0}\left(\tau, x, \alpha_{m}\right)-J_{0}\left(\tau, x, \beta_{m}\right)\right], \\
& R_{1}(\tau, x)=\left(1+\frac{1}{\tau_{s}^{0} \alpha_{m}^{2}}\right)\left[J_{2}(\tau, x, \omega, 0)-J_{2}\left(\tau, x, \omega, \alpha_{m}\right)\right]-\frac{1}{\tau_{s}^{0}}\left[J_{4}(\tau, x, \omega, 0)-J_{4}\left(\tau, x, \omega, \alpha_{m}\right)\right]- \\
& -\frac{1}{\tau_{s}^{0} \alpha_{m}^{2}}\left[J_{2}\left(\tau, x, \omega, \alpha_{m}\right)-J_{2}\left(\tau, x, \omega, \beta_{m}\right)\right], \\
& Q_{2}(\tau, x)=\frac{1}{\omega}\left[J_{0}(\tau, x, 0)-J_{0}\left(\tau, x, \alpha_{m}\right)-J_{3}(\tau, x, \omega, 0)+J_{3}\left(\tau, x, \omega, \alpha_{m}\right)\right]+ \\
& \quad+\frac{\alpha_{m}^{2}}{\left(\alpha_{m}^{4}+\omega^{2}\right)}\left[J_{2}\left(\tau, x, \omega, \alpha_{m}\right)-J_{2}\left(\tau, x, \omega, \beta_{m}\right)\right]+ \\
& \quad+\frac{\omega}{\left(\alpha_{m}^{4}+\omega^{2}\right)}\left[J_{3}\left(\tau, x, \omega, \alpha_{m}\right)-J_{3}\left(\tau, x, \omega, \beta_{m}\right)\right], \\
& R_{2}(\tau, x)=\frac{1}{\omega}\left[J_{2}(\tau, x, \omega, 0)-J_{2}\left(\tau, x, \omega, \alpha_{m}\right)\right]-\frac{1}{2 \omega}\left[J_{2}(\tau, x, 2 \omega, 0)-J_{2}\left(\tau, x, 2 \omega, \alpha_{m}\right)\right]+ \\
& +\frac{\alpha_{m}^{2}}{2\left(\alpha_{m}^{4}+\omega^{2}\right)}\left[J_{0}\left(\tau, x, \alpha_{m}\right)-J_{0}\left(\tau, x, \beta_{m}\right)-J_{3}\left(\tau, x, 2 \omega, \alpha_{m}\right)+J_{3}\left(\tau, x, 2 \omega, \beta_{m}\right)\right]+ \\
& +\frac{\omega}{2\left(\alpha_{m}^{4}+\omega^{2}\right)}\left[J_{2}\left(\tau, x, 2 \omega, \alpha_{m}\right)-J_{2}\left(\tau, x, 2 \omega, \beta_{m}\right)\right], \\
& +\frac{\tau_{m}}{\tau_{m}} . \\
&
\end{aligned}
$$

The functions $J_{k}(\cdot), k=0,1,2,3,4$ in the formulae (7.6)-(7.9) have the form (Prudnikov at al., 1989)

$$
\begin{gathered}
J_{0}(\tau, x, \alpha) \equiv x^{2} e^{-x^{2} \tau} \int_{0}^{\tau} e^{\left(x^{2}-\alpha^{2}\right) s} d s=\frac{x^{2}}{\left(x^{2}-\alpha^{2}\right)}\left(e^{-\alpha^{2} \tau}-e^{-x^{2} \tau}\right), \\
J_{1}(\tau, x, \alpha) \equiv x^{2} e^{-x^{2} \tau} \int_{0}^{\tau} s^{k} e^{\left(x^{2}-\alpha^{2}\right) s} d s=\frac{1}{\left(x^{2}-\alpha^{2}\right)}\left[x^{2} \tau e^{-\alpha^{2} \tau}-J_{0}(\tau, x, \alpha)\right] \\
J_{2}(\tau, x, \omega, \alpha) \equiv x^{2} e^{-x^{2} \tau} \int_{0}^{\tau} e^{\left(x^{2}-\alpha^{2}\right) s} \sin (\omega s) d s= \\
=\frac{x^{2}}{\left[\left(x^{2}-\alpha^{2}\right)^{2}+\omega^{2}\right]}\left\{\left[\left(x^{2}-\alpha^{2}\right) \sin (\omega \tau)-\omega \cos (\omega \tau)\right] e^{-\alpha^{2} \tau}+\omega e^{-x^{2} \tau}\right\},
\end{gathered}
$$




$$
\begin{aligned}
& J_{3}(\tau, x, \omega, \alpha) \equiv x^{2} e^{-x^{2} \tau} \int_{0}^{\tau} e^{\left(x^{2}-\alpha^{2}\right) s} \cos (\omega s) d s= \\
& =\frac{x^{2}}{\left[\left(x^{2}-\alpha^{2}\right)^{2}+\omega^{2}\right]}\left\{\left[\left(x^{2}-\alpha^{2}\right) \cos (\omega \tau)+\omega \sin (\omega \tau)\right] e^{-\alpha^{2} \tau}-\left(x^{2}-\alpha^{2}\right) e^{-x^{2} \tau}\right\} \\
& J_{4}(\tau, x, \omega, \alpha) \equiv x^{2} e^{-x^{2} \tau} \int_{0}^{\tau} s e^{\left(x^{2}-\alpha^{2}\right) s} \sin (\omega s) d s= \\
& =\frac{x^{2}}{\left[\left(x^{2}-\alpha^{2}\right)^{2}+\omega^{2}\right]}\left\{\left[\left(\left(x^{2}-\alpha^{2}\right) \tau-\frac{\left(x^{2}-\alpha^{2}\right)^{2}-\omega^{2}}{\left.\left(x^{2}-\alpha^{2}\right)^{2}+\omega^{2}\right) \sin (\omega \tau)-}\right.\right.\right. \\
& \left.\left.-\left(\omega \tau-\frac{2 \omega\left(x^{2}-\alpha^{2}\right)}{\left(x^{2}-\alpha^{2}\right)^{2}+\omega^{2}}\right) \cos (\omega \tau)\right] e^{-\alpha^{2} \tau}-\frac{2 \omega\left(x^{2}-\alpha^{2}\right)}{\left(x^{2}-\alpha^{2}\right)^{2}+\omega^{2}} e^{-x^{2} \tau}\right\},
\end{aligned}
$$

where the parameter $\alpha \geq 0$.

If the pressure $p^{*}(\tau)$ (2.1) during braking increases monotonically, without oscillations $(a=0)$, then from formulae (7.3) and (7.5) it follows that $P(\tau, x)=Q_{1}(\tau, x)$. Taking the form of functions $Q_{1}(\tau, x)$ (7.6) and $J_{k}(\tau, x, \alpha), k=0,1$ (7.11), (7.12) into account, we obtain

$$
\begin{gathered}
P(\tau, x)=\left(1-e^{-x^{2} \tau}\right)\left(1+\frac{\tau_{m}}{\tau_{s}^{0}}+\frac{1}{\tau_{s}^{0} x^{2}}\right)-\frac{x^{2}\left(e^{-\tau / \tau_{m}}-e^{-x^{2} \tau}\right)}{\left(x^{2}-\tau_{m}^{-1}\right)}\left[1+\frac{2 \tau_{m}}{\tau_{s}^{0}}+\frac{1}{\tau_{s}^{0}\left(x^{2}-\tau_{m}^{-1}\right)}\right]+ \\
+\frac{\tau_{m} x^{2}\left(e^{-2 \tau / \tau_{m}}-e^{-x^{2} \tau}\right)}{\tau_{s}^{0}\left(x^{2}-2 \tau_{m}^{-1}\right)}+\frac{\tau x^{2} e^{-\tau / \tau_{m}}}{\tau_{s}^{0}\left(x^{2}-\tau_{m}^{-1}\right)}-\frac{\tau}{\tau_{s}^{0}}, 0 \leq x<\infty, 0 \leq \tau \leq \tau_{s} .
\end{gathered}
$$

In the limiting case of braking with a constant deceleration at $\tau_{m} \rightarrow 0$ from formula (7.16) we find the results of the Chapter six.

\section{Numerical analysis and conclusion}

Calculations are made for a ceramic-metal pad FMC-11 (the strip) of thickness $d=5 \mathrm{~mm}$ $\left(K_{s}=34.3 \mathrm{Wm}^{-1} \mathrm{~K}^{-1}, \quad k_{s}=15.2 \cdot 10^{-6} \mathrm{~m}^{2} \mathrm{~s}^{-1}\right)$, and a disc (the foundation) from cast iron CHNMKh $\left(K_{f}=51 \mathrm{Wm}^{-1} \mathrm{~K}^{-1}, k_{f}=14 \cdot 10^{-6} \mathrm{~m}^{2} \mathrm{~s}^{-1}\right)$ (Chichinadze at al., 1979). Such a friction pair is used in frictional units of brakes of planes. Time of braking is equal to $t_{s}=3.42 \mathrm{~s}$ $\left(\tau_{s}=2.08\right)$ (Balakin and Sergienko, 1999). Integrals are found by the procedure QAGI from a package of numerical integration QUADPACK (Piessens at al., 1983).

From Chapter six, the results of calculations of dimensionless temperature $\hat{T}^{*}$ (6.2) for the first above considered variants of boundary conditions are presented in Fig. 3a-5a, and for the second - in Fig. 3b-5b. The occurrence of thermal resistance on a surface of contact leads to the occurrence of a jump of temperature on the friction surfaces of the strip and the foundation.

With the beginning of braking, the temperature on a surface of contact $(\zeta=0)$ sharply raises, reaches the maximal value $\hat{T}_{\max }^{*}$ during the moment of time $\tau_{\max }$, then starts to decrease to a minimum level, and finally stops $\tau_{s}$ (Fig. 3a). The heat exchange with an 


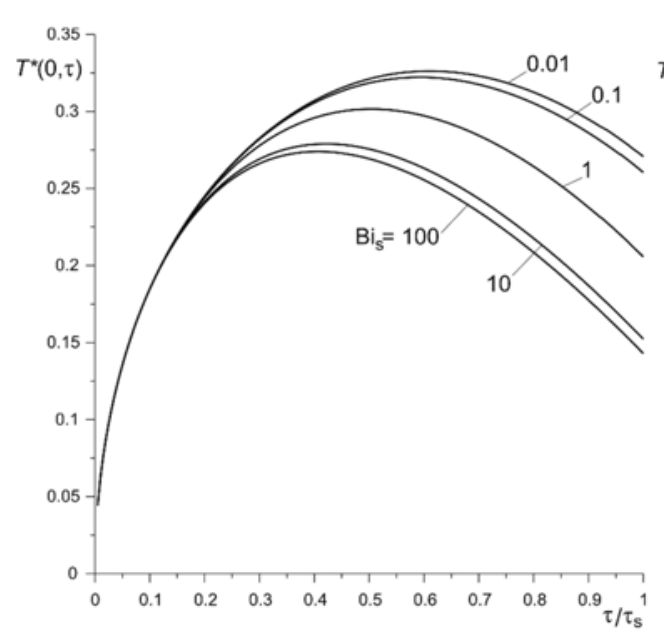

(a)

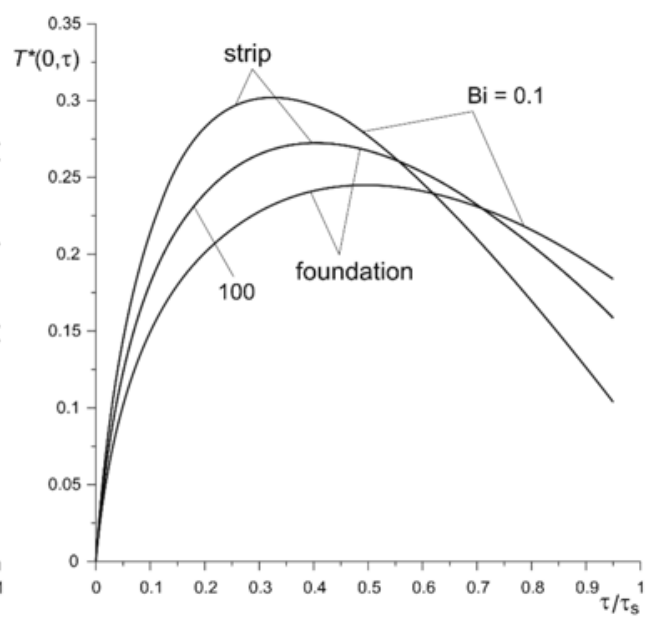

(b)

Fig. 3. Evolution of dimensionless temperature $\hat{T}^{*}$ on a surface of contact $\zeta=0$ for several values of Biot numbers: a) $\mathrm{Bi}_{\mathrm{s}}$; b) Bi , (Yevtushenko and Kuciej, 2010).

environment on an upper surface of a strip does not influence the temperature significantly at an initial stage of braking $0 \leq \tau \leq \tau_{\max }$ when the temperature increases rapidly. This influence is the most appreciable during cooling the surface of contact $\tau_{\max } \leq \tau \leq \tau_{s}$.

When the factor of thermal resistance is small ( $\mathrm{Bi}=0.1)$ the strip is warmed up faster than the foundation, and it reaches the much greater maximal temperature than the maximal temperature on a working surface of the foundation (Fig. 3b). The increase in thermal conductivity of contact area results in alignment of contact temperatures on the friction surface of the bodies. For Biot number $\mathrm{Bi}=100$ the evolutions of temperatures on contact surfaces of the strip and the foundation are identical.

The highest temperature on the surface of contact is reached in case of thermal isolation of the upper surface of the strip $\left(\mathrm{Bi}_{\mathrm{s}} \rightarrow 0\right.$ ) (Fig. 4a). While Biot number increases on the upper surface of the strip, the maximal temperature on surfaces contact decreases. From the data presented in Fig. 4a follows, that for values of Biot number $\mathrm{Bi}_{\mathrm{s}} \geq 20$ to calculate the maximal temperature in considered tribosystem, it is possible to use an analytical solution to a problem, which is more convenient in practice $\left(\mathrm{Bi}_{\mathrm{s}} \rightarrow \infty\right.$ at the set zero temperature on the upper surface of the strip) (Yevtushenko and Kuciej, 2009b).

The effect of alignment of the maximal temperature with increase in thermal conductivity of contact surfaces is especially visible in Fig. $4 \mathrm{~b}$. To calculate the maximal temperature at $\mathrm{Bi} \geq 10$, we may use formulas (6.2)-(6.5), which present the solutions to the thermal problem of friction at braking in case of an ideal thermal contact of the strip and the foundation, and of maintenance of zero temperature on the upper surface of the strip.

Change of dimensionless temperature in the strip and the foundation on a normal to a friction surface for Fourier's number $\tau_{s}=2.08$ is shown in Fig. 5. The temperature reaches the maximal value on the friction surface $\zeta=0$, and decreases while the distance from it grows. The drop of temperature in the strip for small values of Biot number $\left(\mathrm{Bi}_{\mathrm{s}}=0.1\right)$ has nonlinear character (Fig. 5a). If the zero temperature is maintained $\left(\mathrm{Bi}_{\mathrm{s}}=100\right)$ during 


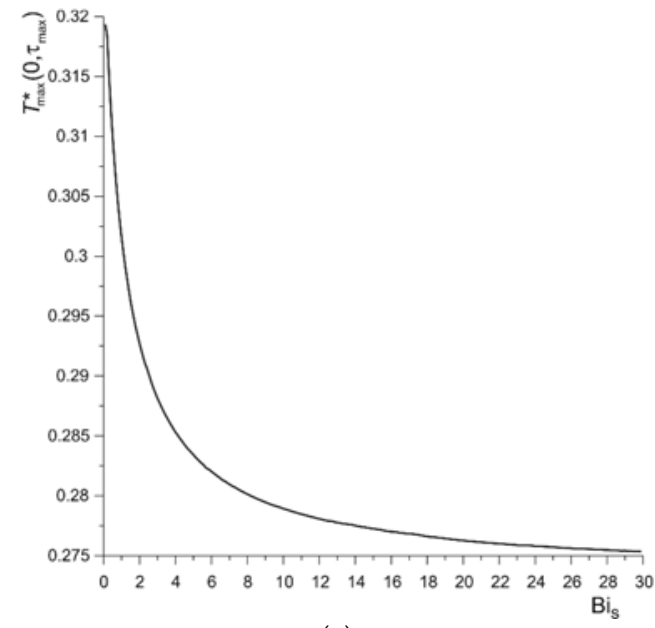

(a)

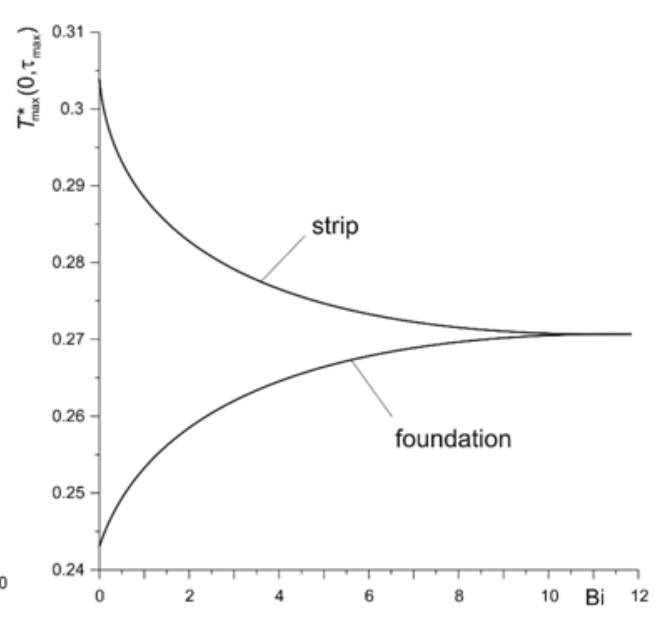

(b)

Fig. 4. Dependence of dimensionless maximal temperature $\hat{T}_{\max }^{*}$ on Biot numbers: a) $\mathrm{Bi}_{s}$;

b) Bi for dimensionless time of braking $\tau_{s}=2.08$, (Yevtushenko and Kuciej, 2010).

braking on the upper surface of the strip, then the reduction of temperature in the strip, and while the distance from the friction surface grows, can be described by a linear function of dimensionless spatial variable $\zeta$. The effective depth of heating up the foundation decreases with the increase in Biot number and for values $\mathrm{Bi}_{\mathrm{s}}=0.1 ; 100$ is equal 2.4 and 2.15 of the strip's thickness accordingly. Irrespective of size of thermal resistance, the temperature in the strip linearly decreases from the maximal value for surfaces of contact up to zero on the upper surface of the strip (Fig. 5b). The effective depth of heating up the foundation increases with the increase of thermal resistance (reduction of thermal conductivity) - for values $B i=0.1 ; 100$ it is equal 2.15 and 2.7 of thickness of the strip accordingly.

From Chapter seven, the results of calculations of dimensionless temperature $\hat{T}^{*}$ (7.1) are presented in Figs. 5-7. First, for fixed values of the input parameters $\tau_{m}, \tau_{s}^{0}, a$ and $\omega$ we find numerically the dimensionless time of stop $\tau_{s}$ as the root of functional equation (2.9). Knowing the time of braking $\tau_{s}$, we can construct the dependencies of output parameters on the ratio $\tau / \tau_{s}$. Such dependencies for the dimensionless pressure $p^{*}$ (2.1) and sliding speed $V^{*}$ (2.4) are shown in Fig. 6. We see in Fig. 6a four curves for two values of the dimensionless time of pressure rise, which corresponds to instantaneous $\left(\tau_{m}=0\right)$ and monotonic $\left(\tau_{m}=0.2\right)$ increase in pressure to the nominal value, at two values of the amplitude $a=0$ and $a=0.1$. In Fig. $6 \mathrm{~b}$ we see only two curves constructed at the same values of parameters $\tau_{m}$ and $a$. This is explained by the fact that the amplitude of fluctuations of pressure $a$ practically does not influence the evolution of speed of sliding.

The evolution of the dimensionless contact temperature $\hat{T}^{*}(0, \tau)(7.2)$ in the pad and in the disc, for the same distributions of dimensionless pressure $p^{*}(2.1)$ and velocity $V^{*}(2.4)$, which are shown in Figs. 6a,b is presented in Fig. 7. Due to heat transfer through the surface of contact the temperatures of the pad (Fig. 7a) and the disk (Fig. 7b) on this surface are various. The largest value of the contact temperature is reached during braking with the 


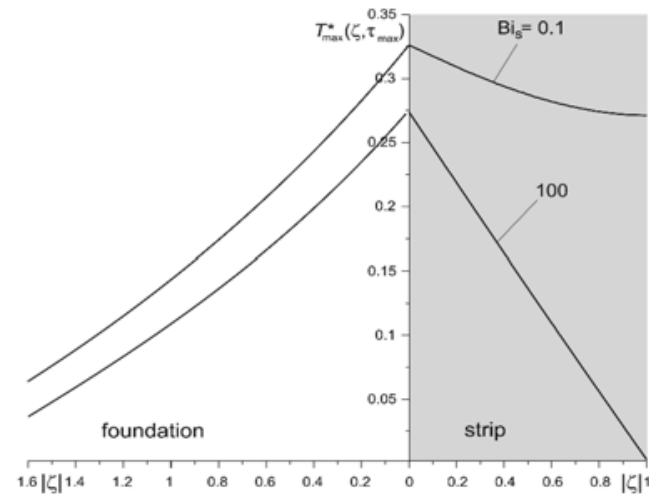

(a)

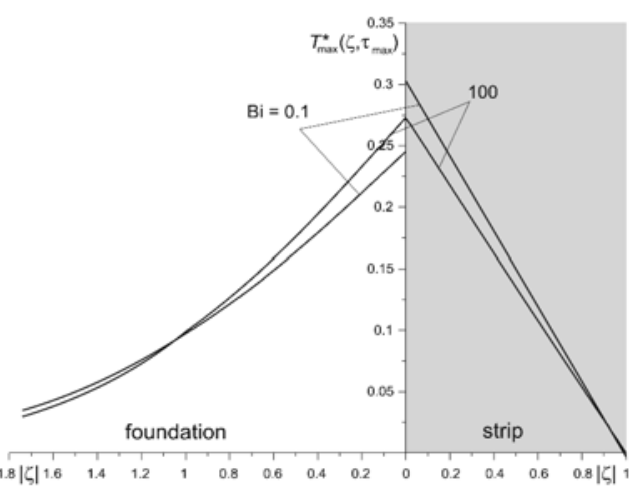

(b)

Fig. 5. Distribution of dimensionless temperature $\hat{T}^{*}$ in the strip $(0 \leq \zeta \leq 1)$ and the foundation $(-\infty \leq \zeta \leq 0)$ during the dimensionless moment of time $\tau=\tau_{\max }$ of reaching the temperature of the maximal value $T_{\max }^{*}$ for two values of Biot numbers: a) $\mathrm{Bi}_{\mathrm{s}}$; b) Bi, (Yevtushenko and Kuciej, 2010).

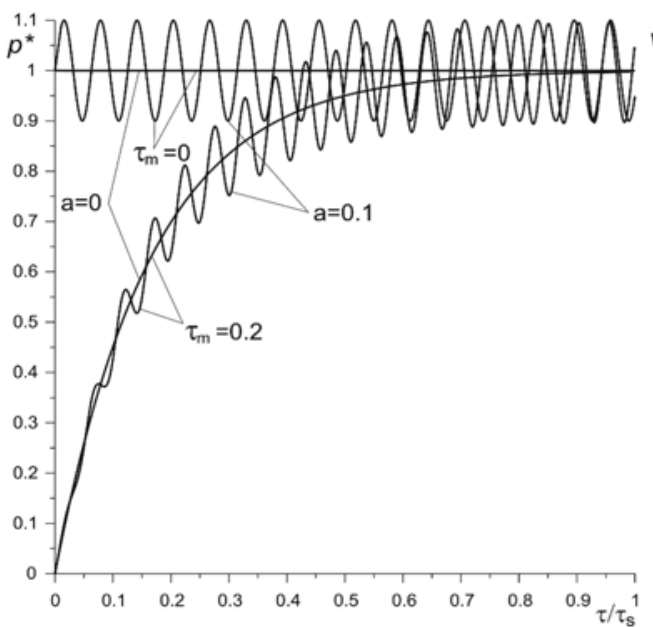

(a)

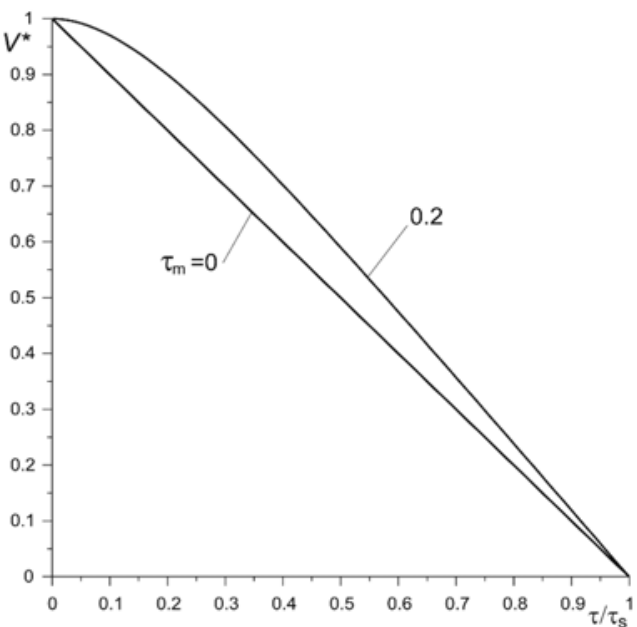

(b)

Fig. 6. Evolution of the dimensionless pressure $p^{*}$ (a) and sliding speed $V^{*}$ (b) during braking for several values of the Fourier number $\tau_{m}$ and dimensionless amplitude $a$, (Yevtushenko at al. 2010).

constant deceleration $\left(\tau_{m}=0\right)$. The increase in duration of achieving the nominal value of pressure leads to a decrease in contact temperature. The maximum contact temperature in the case of braking with the constant deceleration $\left(\tau_{m}=0\right)$ is always larger than at the nonuniform braking. It is interesting, that the temperature at the moment of a stop is practically independent of the value of the parameter $\tau_{m}$. Pressure oscillations (see Fig. 6) lead to the fact that the temperature on the contact surface also oscillates, but with a considerably lower amplitude (Fig. 7). 


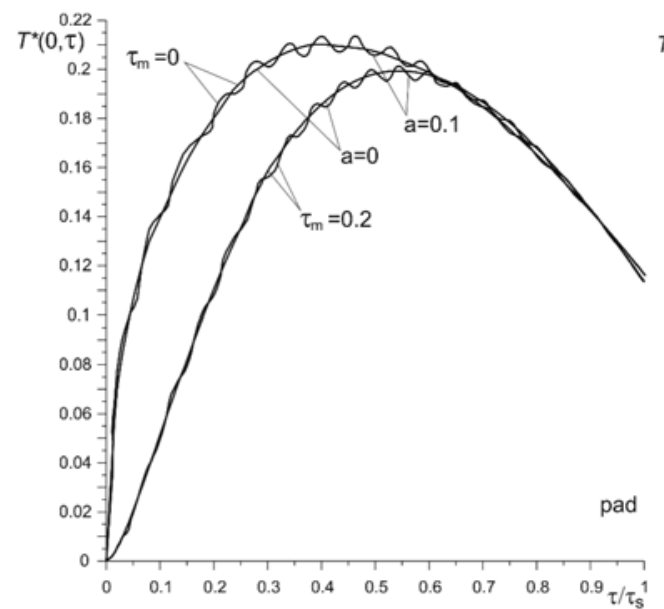

(a)

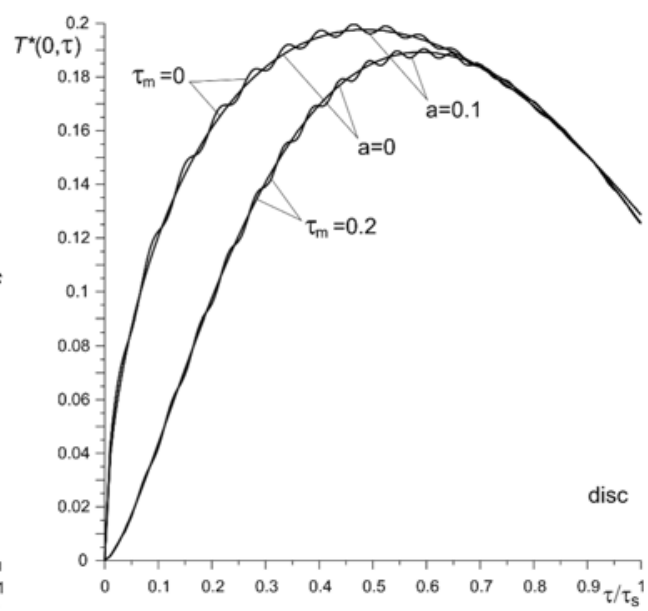

(b)

Fig. 7. Evolution of dimensionless temperature $\hat{T}^{*}(0, \tau)(7.2)$ on the contact surface of the pad (a) and the disc (b) for two values of the Fourier number $\tau_{m}=0 ; 0.2$ and dimensionless amplitude $a=0 ; 0.1$ at fixed values of the dimensionless input parameters $\tau_{s}^{0}=1, \mathrm{Bi}=5$, (Yevtushenko at al. 2010).

Evolution of dimensionless temperature $\hat{T}^{*}(\zeta, \tau)$ not only on a surface of contact, but also inside the pad and the disc is shown in Fig. 8. Regardless of the value of the time of pressure increase, the temperature oscillations take place in a thin subsurface layer. The thickness of this layer is about 0.2 of the thickness of the pad. Also, in these figures we see "the effect of delay" - the moment of time of achieving the temperature of the maximal value increases with the increase in distance from a surface of friction. In the pad the maximum temperature is reached before stopping at a given distance from the friction surface (Figs. 8a,c). In the disc we observe a different picture - for a depth $\geq 0.6 d$ the temperature reaches a maximum value at the stop time moment (Figs. $8 \mathrm{~b}, \mathrm{~d}$ ).

\section{Conclusions}

The analytical solutions to a thermal problem of friction during braking are obtained for a plane-parallel strip/semi-space tribosystem with a constant or time-dependence friction power. In the solutions we take into account the heat transfer through a contact surface, and convective exchange on the upper surface of the pad. To solve the thermal problem of friction with time-dependent friction power we use solution to thermal problem with constant friction power and Duhamel formula (6.1).

The investigation is conducted for ceramic-metal pad (FMC-11) and cast iron disc (CHNMKh). The results of our investigation of the frictional heat generation of the pad sliding on the surface of the disc in the process of braking allow us to make the main conclusions, i.e. the temperature on the contact surface rises sharply with the beginning of braking, and at about half braking time it reaches the maximal value. Then, till the moment of stopping, the fall of temperature occurs (Fig. 3); the increase of convective exchange $\left(\mathrm{Bi}_{\mathrm{s}}\right)$ on the outer surface of the pad, leads to the decrease of the maximal temperature on the 


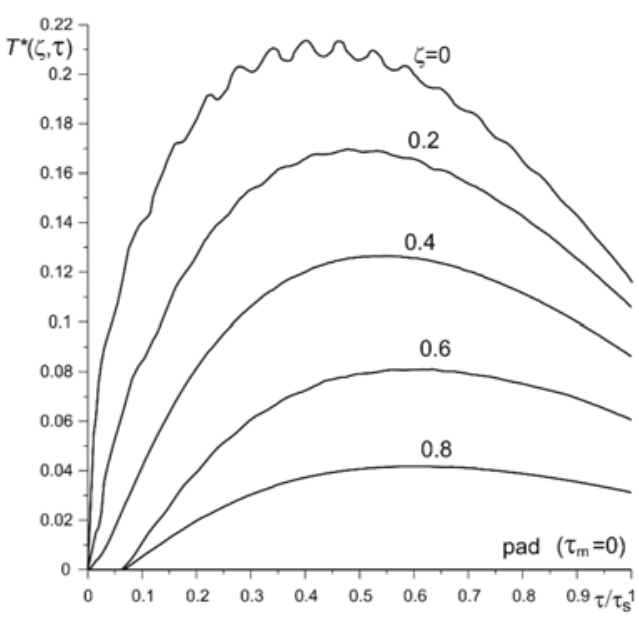

(a)

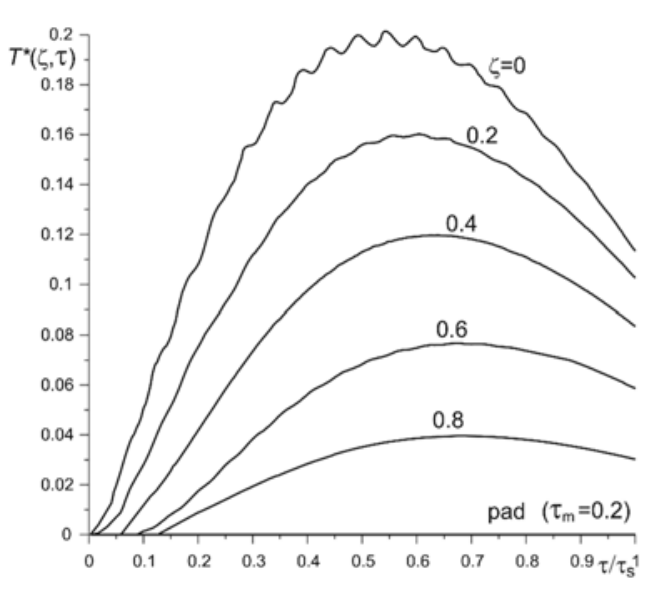

(c)

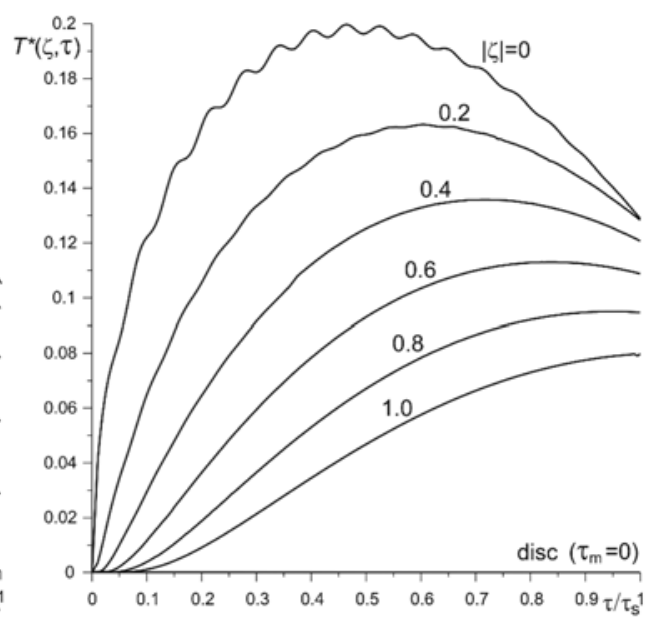

(b)

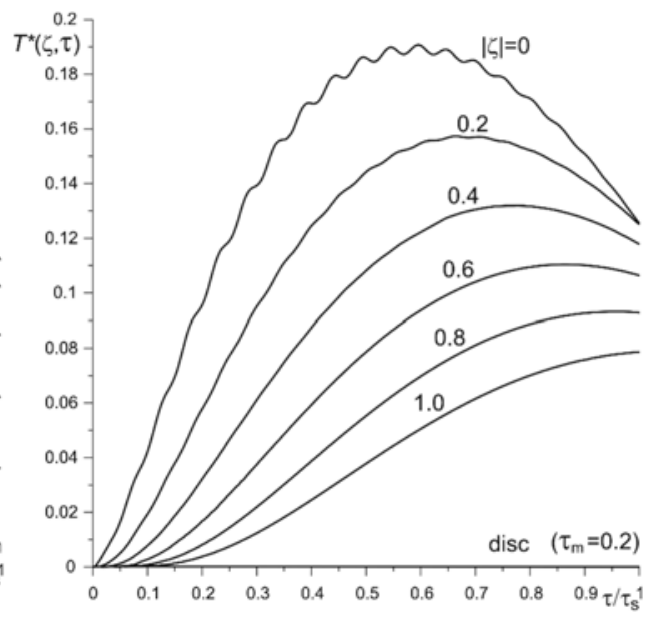

(d)

Fig. 8. Evolution of dimensionless temperature $\hat{T}^{*}(\zeta, \tau)(7.2)$ in the pad (a), (c) and in the disc (b), (d) for two values of the of the Fourier number $\tau_{m}=0$ (a), (b) and $\tau_{m}=0.2$ (c), (d) at fixed values of the dimensionless input parameters $a=0.1, \tau_{s}^{0}=1, \mathrm{Bi}=5$, (Yevtushenko at al. 2010).

contact surface, while the time of reaching it gets shorter (Fig. 4a); the reduction of the thermal resistance on the contact surface (the increase of Biot's number Bi) causes the equalization of the maximal temperatures of the pad and disc's surfaces and of the time of reaching it (Fig. 4b); that the contact temperature decreases with the increase in dimensionless input parameter $\tau_{m}$ (duration increase in pressure from zero to the nominal value) (Figs. 7); the amplitude of the oscillations of temperature is much less than the amplitude of corresponding fluctuations of pressure ("the leveling effect") (Figs. 7, 8). 


\section{References}

Abramovits, M., Stegun, I. (1979). Handbook of Mathematical Functions, 2 nd. edn, Dover, New York.

Archard, J. F., Rowntree, R. A. (1988). The temperature of rubbing bodies; Part 2, The distribution of temperatures, Wear, Vol. 128, pp. 1-17.

Balakin, V.A., Sergienko, V.P. (1999). Heat calculations of brakes and friction units, MPRI of NASB, Gomel (in Russian).

Barber, J. R., Comminou, M. (1989). Thermoelastic contact problems. In: Thermal Stresses III. Ed. R.R. Hetnarsky, Elsevier Sci. Publ., pp.1-105.

Bateman, H., Erdelyi, A. (1954). Tables of Integral Transforms, V. 1, McGraw-Hill, New York.

Bauzin, J. G., Laraqi, N. (2004). Simultaneous estimation of frictional heat flux and two thermal contact parameters for sliding contacts, Numerical Heat Transfer; Part A: Applications, Vol. 45, No 4, pp. 313-328.

Block, H. (1937). Theoretical studies of temperature rise at surfaces of actual contact under oiliness lubrication conditions, Proc. General Discussion on Lubrication and Lubricants. Institute of Mechanical Engineers, Vol. 2, pp. 222-235.

Burton, R. A. (1975). An axisymmetric contact patch configuration for two slabs in frictionally heated contact, The mechanics of the contact between deformable bodies, Proc. IUTAM Symp., ed. de Pater A. D., Kalker J. J., Delft Univ. Press.

Cameron, A., Gordon, A.N., Symm, G.T. (1965). Contact temperatures in rolling/sliding surfaces, Proc. Roy. Soc., A, Vol. 286, pp. 45-61.

Carslaw, H.S., Jaeger, J.C. (1959). Conduction of Heat in Solids, Clarendon Press, Oxford.

Chichinadze, A.V. (1967). Calculation and investigation of external friction during braking, Nauka, Moscow (in Russian).

Chichinadze, A.V., Braun, E.D., Ginsburg, A.G., et al., (1979). Calculation, test and selection of frictional couples, Nauka, Moscow (in Russian).

Evtushenko, A., Matysiak, S., Kutsei, M., (2005). Thermal problem of friction at braking of coated body, J. Friction and Wear, Vol. 26, No 2, pp. 33-40.

Evtushenko, O.O., Pyr'ev, Yu.O. (2000). Temperature and wear of the friction surfaces of a cermets patch and metal disk in the process of braking, Materials Science, Vol. 36, No 2, pp. 218-223.

Fasekas, G. A. G. (1953). Temperature gradients and heat stresses in brake drums, SAE Trans., Vol. 61, pp. 279-284.

Ginsburg, A.G., Chichinadze, A.V. (1978). Complex estimation of performance data of frictional brakes on a design stage, In: Problems of non-stationary friction in machines, devices and apparatus, Nauka, Moscow, pp. 10-43 (in Russian).

Godet, M. (1990). Third-bodies in tribology, Wear, Vol. 136, No 1, pp. 29-45.

Grylytskyy, D.V. (1996) Thermoelastic contact problems in tribology, Institute of the Maintenance and Methods of Training of the Ministry of Education of Ukraine, Kiev (in Ukrainian).

Ho, T.L., Peterson, M.B., Ling, F.F. (1974). Effect of frictional heating on braking materials, Wear, Vol. 26, pp. 73-79.

Iordanoff, I., Berthier, Y., Descartes, S., Heshmat, H. (2002). A review of recent approaches for modeling solid third bodies, Trans. ASME, J. Tribology, Vol. 124, No 4, pp. 725735 . 
Jaeger, J. C. (1942). Moving sources of heat and the temperatures of sliding contacts, Proc. Roy. Soc. New South Wales, Vol. 76, No 3, pp. 203-224.

Johnson, K. L., (1987). Contact Mechanics, Cambridge Univ. Press, Cambridge.

Kahveci, K., Can, Y., Cihan, A. (2005). Heat transfer in continuous-drive friction welding of different diameters, Numerical Heat Transfer; Part A, Vol. 48, No 10, pp. 1035-1050.

Kannel, J. W., Barber, S. A. (1989). Estimate of surface temperatures during rolling contact, Tribology Trans., Vol. 32, No 3, pp. 305-310.

Levitskij, V.P., Onyshkievich, V.M. (1999). Investigation of influence of "third body" properties on heat generation due to friction, Math. Methods Phys. Mech. Fields, Vol. 42 , pp. $82-86$.

Ling, F. F. (1973). Surface Mechanics, Wiley, New York.

Ling, F. F., (1959). A quasi-iterative method for computing interface temperature distributions, Zeitschrift für angewandte Mathematik und Physik (ZAMP), Vol. 10, No 5, pp. 461-474.

Luikov, A.V. (1968). Analytical heat diffusion theory, Academic Press, New York.

Matysiak, S., Evtushenko A., Kutsei, M. (2004). Non-stationary heating of a uniform foundation coated with a surface composite layer, J. Friction and Wear, Vol. 25, No 6, pp. 11-18.

Matysiak, S., Konieczny, S., Yevtushenko, A. (1998). Distribution of friction heat during cold-rolling of metals by using composite rolls, Numerical Heat Transfer; Part A, Vol. 34, No 7, pp. 719-729.

Matysiak, S., Yevtushenko, O., Kutsiei, M. (2007). Temperature field in the process of braking of a massive body with composite coating, Materials Science, Vol. 43, pp. 62-69.

Naji, M., Al-Nimr, M. (2001). Dynamic thermal behavior of a brake system, Int. Comm. Heat Mass Trans., Vol. 28, pp. 835-845.

Nosko, A.L., Nosko, A.P. (2006). Solution of contact heating problem with account for heat transfer between the friction members, J. Friction and Wear, Vol. 27, pp. 35-40.

Olesiak, Z., Pyryev, Yu., Yevtushenko, A. (1997). Determination of temperature and wear during braking, Wear, Vol. 210, pp. 120-126.

Piessens, R., E. de Doncker-Kapenga, Überhuber, C.W., Kahaner, D.K. (1983). QUADPACK: A Subroutine Package for Automatic Integration, Springer-Verlag, Berlin.

Prudnikov, A.P., Brychkov, Yu. A., Marychev, O.I. (1981). Integrals and Series, Nauka, Moscow (in Russian).

Pyryev, Yu., Yevtushenko, A. (2000). The influence of the brakes friction elements thickness on the contact temperature and wear, Heat and Mass Transfer, Vol. 36, pp. 319-323.

Sneddon, I. N. (1972). The Use of Integral Transforms, McGraw-Hill, New York.

Yevtushenko, A. A., Ivanyk, E. G., Sykora, O. V. (1995). The transitive temperature processes in local friction contact, Int. J. Heat Mass Transfer, Vol. 38, No 13, pp. 2395-2401.

Yevtushenko, A., Kuciej, M. (2009a). Influence of the protective strip properties on distribution of the temperature at transient frictional heating, Int. J. Heat Mass Transfer, Vol. 52, No 1/2, pp. 376-384.

Yevtushenko, A.A., Kuciej, M. (2009b). Two contact problems for the strip with the frictional heating during braking, Proc. 8th Int. Congr. on Thermal Stresses, TS2009, UrbanaChampaign (Illinois, USA), June 1-4; pp. 289-293. 
Yevtushenko, A., Kuciej, M. (2010). Influence of the convective cooling and the thermal resistance on the temperature of the pad/disc tribosystem, Int. Comm. Heat Mass Transfer, Vol. 37, pp. 337-342.

Yevtushenko, A., Kuciej, M. Yevtushenko, O. (2010). Influence of the pressure fluctuations on the temperature in pad/disc tribosystem, Int. Comm. Heat Mass Transfer, Vol. 37, pp. 978-983.

Yevtushenko, A., Kuciej, M., Rożniakowska, M. (2005). Thermal cleavage stresses in a piecewise-homogeneous plate, Materials Science, Vol. 5, pp. 581-588.

Yevtushenko, A. A., Rozniakowska, M., Kuciej, M. (2007a). Transient temperature processes in composite strip and homogeneous foundation, Int. Comm. Heat Mass Transfer, Vol. 34, pp. 1108-1118.

Yevtushenko, A., Rożniakowska, M., Kuciej, M. (2007b). Laser-induced thermal splitting in homogeneous body with coating, Numerical Heat Transfer, Part. A, Vol. 52, pp. 357375 .

Yevtushenko, A., Tolstoj-Sienkiewicz J. (2006). Temperature in a rotating ring subject to frictional heating from two stationary pins, Numerical Heat Transfer; Part A, Vol. 49, No 8, pp. 785-801.

Yevtushenko, A.A., Ivanyk, E.G., Yevtushenko, O.O. (1999) Exact formulae for determination of the mean temperature and wear during braking, Heat and Mass Transfer, Vol. 35, pp. 163-169.

Yi, YBo, Barber, J.R., Hartsock, D.L. (2002). Thermoelastic instabilities in automotive disc brakes - finite element analysis and experimental verification, In: Martins JAC and Monteiro Marques MDP, editors. Contact Mechanics, Kluwer, Dordrecht, p. 187202. 


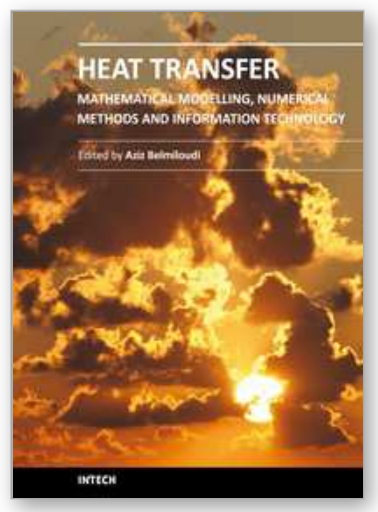

\section{Heat Transfer - Mathematical Modelling, Numerical Methods and Information Technology}

Edited by Prof. Aziz Belmiloudi

ISBN 978-953-307-550-1

Hard cover, 642 pages

Publisher InTech

Published online 14, February, 2011

Published in print edition February, 2011

Over the past few decades there has been a prolific increase in research and development in area of heat transfer, heat exchangers and their associated technologies. This book is a collection of current research in the above mentioned areas and describes modelling, numerical methods, simulation and information technology with modern ideas and methods to analyse and enhance heat transfer for single and multiphase systems. The topics considered include various basic concepts of heat transfer, the fundamental modes of heat transfer (namely conduction, convection and radiation), thermophysical properties, computational methodologies, control, stabilization and optimization problems, condensation, boiling and freezing, with many real-world problems and important modern applications. The book is divided in four sections : "Inverse, Stabilization and Optimization Problems", "Numerical Methods and Calculations", "Heat Transfer in Mini/Micro Systems", "Energy Transfer and Solid Materials", and each section discusses various issues, methods and applications in accordance with the subjects. The combination of fundamental approach with many important practical applications of current interest will make this book of interest to researchers, scientists, engineers and graduate students in many disciplines, who make use of mathematical modelling, inverse problems, implementation of recently developed numerical methods in this multidisciplinary field as well as to experimental and theoretical researchers in the field of heat and mass transfer.

\section{How to reference}

In order to correctly reference this scholarly work, feel free to copy and paste the following:

Aleksander Yevtushenko and Michal Kuciej (2011). Frictional Heating in the Strip-Foundation Tribosystem, Heat Transfer - Mathematical Modelling, Numerical Methods and Information Technology, Prof. Aziz Belmiloudi (Ed.), ISBN: 978-953-307-550-1, InTech, Available from: http://www.intechopen.com/books/heattransfer-mathematical-modelling-numerical-methods-and-information-technology/frictional-heating-in-the-stripfoundation-tribosystem

\section{INTECH}

open science | open minds

\section{InTech Europe}

University Campus STeP Ri

Slavka Krautzeka 83/A

51000 Rijeka, Croatia

Phone: +385 (51) 770447

\section{InTech China}

Unit 405, Office Block, Hotel Equatorial Shanghai

No.65, Yan An Road (West), Shanghai, 200040, China

中国上海市延安西路65号上海国际贵都大饭店办公楼 405 单元

Phone: +86-21-62489820 
Fax: +385 (51) 686166

Fax: +86-21-62489821

www.intechopen.com 
(C) 2011 The Author(s). Licensee IntechOpen. This chapter is distributed under the terms of the Creative Commons Attribution-NonCommercialShareAlike-3.0 License, which permits use, distribution and reproduction for non-commercial purposes, provided the original is properly cited and derivative works building on this content are distributed under the same license. 